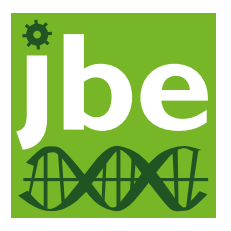

JOURNAL OF BIOLOGICAL ENGINEERING

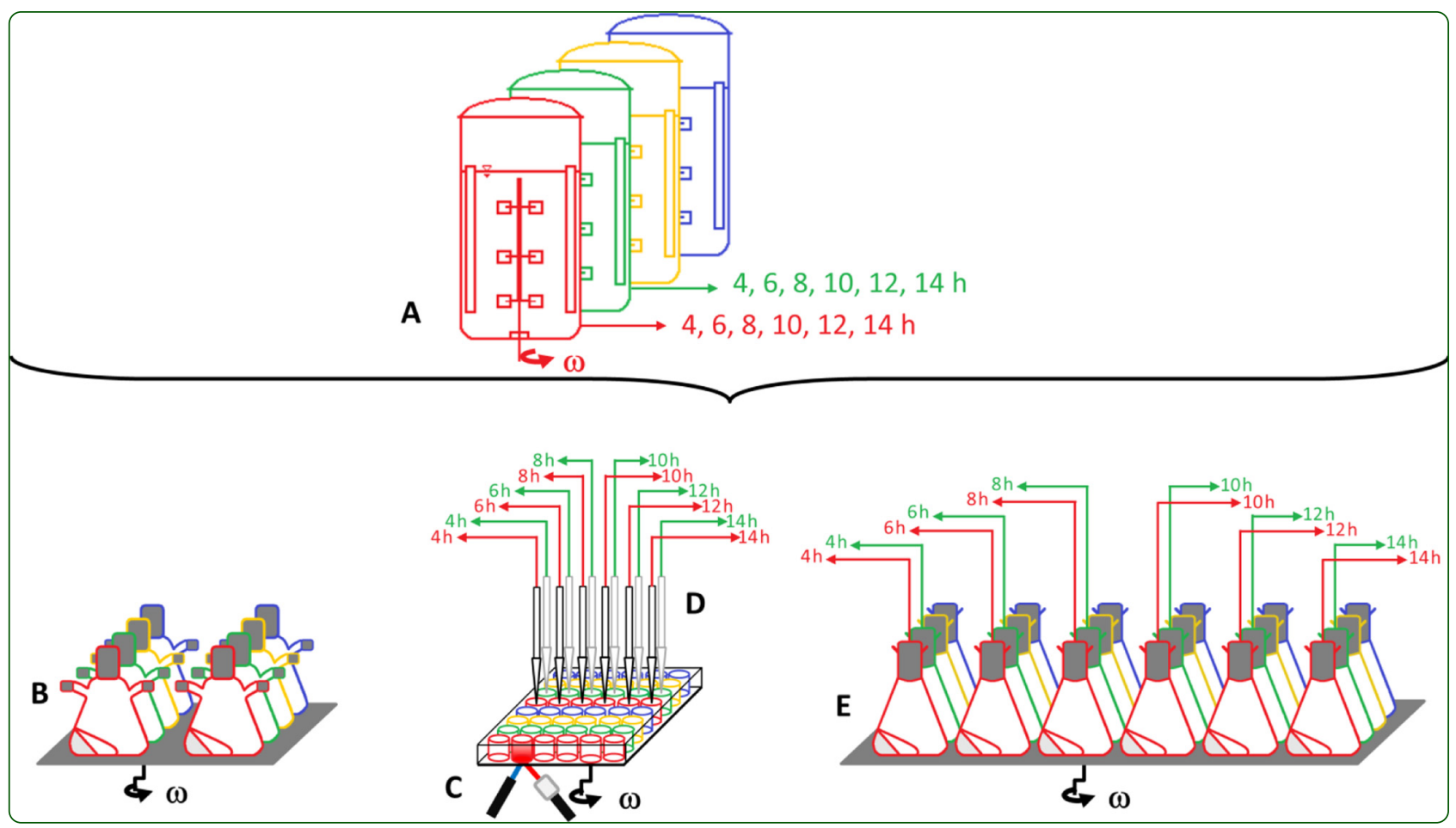

Parallel use of shake flask and microtiter plate online measuring devices (RAMOS and BioLector) reduces the number of experiments in laboratory-scale stirred tank bioreactors

Wewetzer et al. 


\title{
Parallel use of shake flask and microtiter plate online measuring devices (RAMOS and BioLector) reduces the number of experiments in laboratory-scale stirred tank bioreactors
}

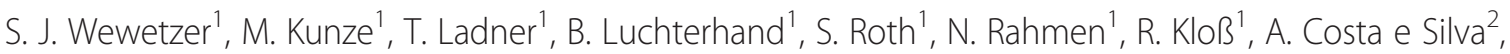

L. Regestein ${ }^{1}$ and J. Büchs ${ }^{1 *}$

\begin{abstract}
Background: Conventional experiments in small scale are often performed in a 'Black Box' fashion, analyzing only the product concentration in the final sample. Online monitoring of relevant process characteristics and parameters such as substrate limitation, product inhibition and oxygen supply is lacking. Therefore, fully equipped laboratoryscale stirred tank bioreactors are hitherto required for detailed studies of new microbial systems. However, they are too spacious, laborious and expensive to be operated in larger number in parallel. Thus, the aim of this study is to present a new experimental approach to obtain dense quantitative process information by parallel use of two small-scale culture systems with online monitoring capabilities: Respiration Activity MOnitoring System (RAMOS) and the BioLector device.

Results: The same 'mastermix' (medium plus microorganisms) was distributed to the different small-scale culture systems: 1) RAMOS device; 2) 48-well microtiter plate for BioLector device; and 3) separate shake flasks or microtiter plates for offline sampling. By adjusting the same maximum oxygen transfer capacity (OTR $R_{\max }$ ), the results from the RAMOS and BioLector online monitoring systems supplemented each other very well for all studied microbial systems (E. coli, G. oxydans, K. lactis) and culture conditions (oxygen limitation, diauxic growth, auto-induction, buffer effects).

Conclusions: The parallel use of RAMOS and BioLector devices is a suitable and fast approach to gain comprehensive quantitative data about growth and production behavior of the evaluated microorganisms. These acquired data largely reduce the necessary number of experiments in laboratory-scale stirred tank bioreactors for basic process development. Thus, much more quantitative information is obtained in parallel in shorter time.
\end{abstract}

Keywords: Shake flasks, Microtiter plates, RAMOS, BioLector, RoboLector, Online measurement, Parallel cultivation, Small-scale cultivation, Oxygen limitation, High-throughput

\section{Background}

In recent years, there has been a constantly increasing demand for high-throughput experimentation in biotechnological applications. Very interesting approaches have been proposed for designing miniature stirred bioreactors with culture volumes as low as $50 \mu \mathrm{L}$ [1-7]. Yet, microtiter plates (MTP) and shake flasks remain the

\footnotetext{
*Correspondence: Jochen.Buechs@avt.rwth-aachen.de

${ }^{1}$ RWTH Aachen University, AVT - Biochemical Engineering, Worringer Weg 1, 52074 Aachen, Germany

Full list of author information is available at the end of the article
}

most commonly used small-scale culture systems that are often applied especially for high-throughput screening [8] and in the early stages of bioprocess development. MTPs are most often used for high-throughput applications, whereas shake flask cultivations are first choice for follow-up experiments. Numerous studies have been conducted to evaluate the performance of a microbial strain under different experimental conditions in order to determine the best suitable candidate for a given process. In both MTP and shake flask systems, however, only little (quantitative) data are gained. Moreover, it 
is very challenging to perform experiments under controlled fermentation conditions. Therefore, the optimization of process parameters is performed in laboratory-scale stirred tank bioreactors. However, bioreactor size restricts the number of parallel experiments that can be conducted. The sequence, starting at strain construction and ending with pilot-scale trials (compare Fig. 1), results in long development times, termed as a "resource burden" by Bareither and Pollard in 2011 [1]. Furthermore, this whole process may lead to false selection of strains and media due to insufficient data and overlooked unsuitable operating conditions in MTPs and shake flasks. These wrong selections would later adversely affect the whole production process [9]. Consequently, small-scale online monitoring systems such as the Respiration Activity MOnitoring System (RAMOS) and the BioLector devices have been developed [10-16].

With the microtiter plate-based BioLector platform important online information can be optically generated on biomass formation, $\mathrm{pH}$-value and dissolved oxygen tension (DOT) as well as of fluorescence either of respective metabolites (e.g. tryptophan, $\mathrm{NADH}$ or riboflavin) or of target proteins eventually tagged by fluorescent marker proteins. Numerous studies have been carried out experimentally proving the value of this highthroughput device [15, 17-20]. However, there is the drawback that the scattered light and the fluorescence signals only provide information relative to a reference and have to be calibrated to obtain quantitative absolute values [21].

As described by Kensy et al., a linear correlation between scattered light and biomass could be obtained to $50 \mathrm{~g} / \mathrm{L}$ of cell dry weight [18]. Respiration activity available from a stirred tank bioreactor equipped with an exhaust gas analyzer is not provided by the BioLector device. Exactly this missing information can be generated by the RAMOS device $[12,13]$ on shake flask level. This device measures online the oxygen and carbon dioxide evolution rates in eight parallel shake flasks without the need for calibration. Correct absolute values are provided which can for example also be used to calculate material balances and stoichiometries.

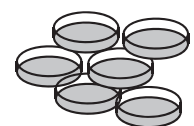

Strain construction $\mathrm{n} \approx 10,000$

(1)

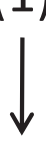

(2)

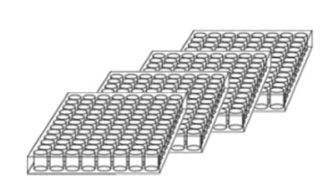

Primary strain screening

$\mathrm{n} \approx 10,000$

$0.05-1 \mathrm{~mL}$

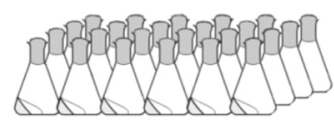

Secondary strain screening $\mathrm{n} \approx 50-100$ $2-100 \mathrm{~mL}$

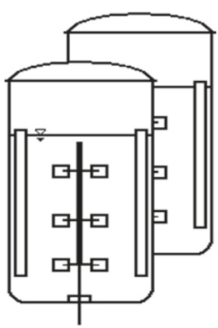

Initial process development

$\mathrm{n} \approx 2-10$

$2-10 L$
(4)
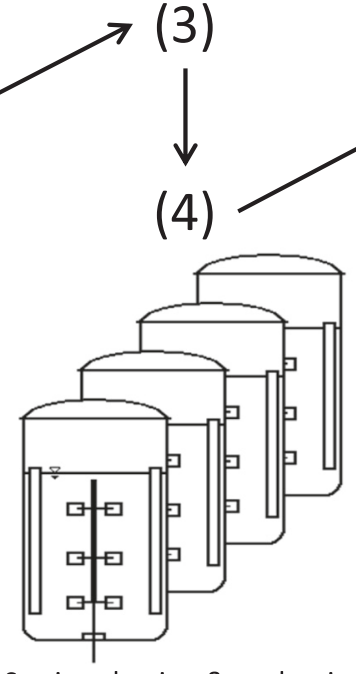

Strain selection \& evaluation

$\mathrm{n} \approx 10-20$

$0.5-5 \mathrm{~L}$
(5)

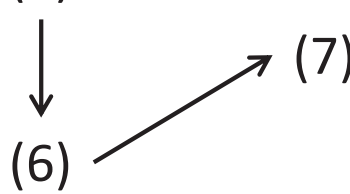

(7)

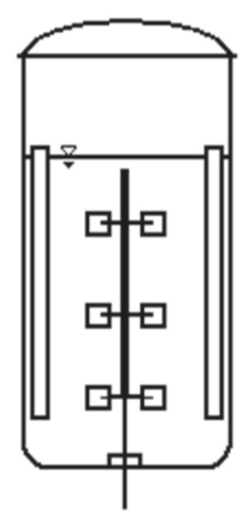

Pilot-scale trials

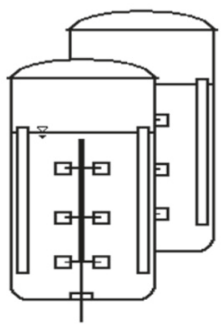

Process optimization

$$
\mathrm{n} \approx 1-4
$$$$
10-100 \mathrm{~L}
$$

Fig. 1 Scheme of an exemplary bioprocess development chain. This scheme represents a conventional development chain of a bioprocess from strain construction to pilot-scale (adopted from Figure one of Bareither and Pollard [1]) 
The aim of this current study is to present a new efficient experimental approach (Fig. 2) combining a RAMOS (Fig. 2b) and a BioLector device (Fig. 2c) coupled with an automated liquid handling system (Fig. 2d) or separate shake flasks (Fig. 2e) for offline sampling to reduce the necessary number of experiments in laboratory-scale stirred tank bioreactor (Fig. 2a). Especially we are focusing on step (4) and (5) of a conventional process development chain as depicted in Fig. 1. By coupling the BioLector device with an automated liquid handling system, in whole termed RoboLector (Fig. 2d) [17], automated sampling can be realized, replacing separate shake flasks if only small sample volumes (about $1 \mathrm{~mL}$ ) are required for analysis. Each separate shake flask or sampling well is withdrawn at specific time points and not reused for later sampling in order to avoid the modification of the well or flask filling volume [22]. Figure 2 presents four experimental variants each coded with a different color. While for each experimental variant one stirred tank bioreactor would be needed, all four variants can be run simultaneously in one RAMOS device in duplicate.
For successful application of the proposed new experimental approach, all bioreactors should be operated at essentially equivalent conditions in order to be considered as one experiment, obtained from one theoretical reactor. Only if this requirement is assured, the microorganisms will behave in an equivalent way. For that goal, the key engineering parameter has to be identified for each microbial system. In this work, the oxygen supply was chosen as the key parameter and, therefore, the maximum oxygen transfer capacity $\left(\mathrm{OTR}_{\max }\right)$ in the different bioreactors was kept constant. If the $\mathrm{OTR}_{\max }$ is constant, then the results from different bioreactors operated in parallel can be combined and regarded as one experiment, similar to the results obtained from one single laboratory-scale stirred tank bioreactor (see Fig. 2). The same starting conditions must be used, because slight differences in the initial optical density $\left(\mathrm{OD}_{600}\right)$ can cause a significant difference in the duration of the lag-phase [17]. Identical starting conditions are achieved by inoculating the total amount of medium (mastermix) required for one experiment. Then, the mastermix is

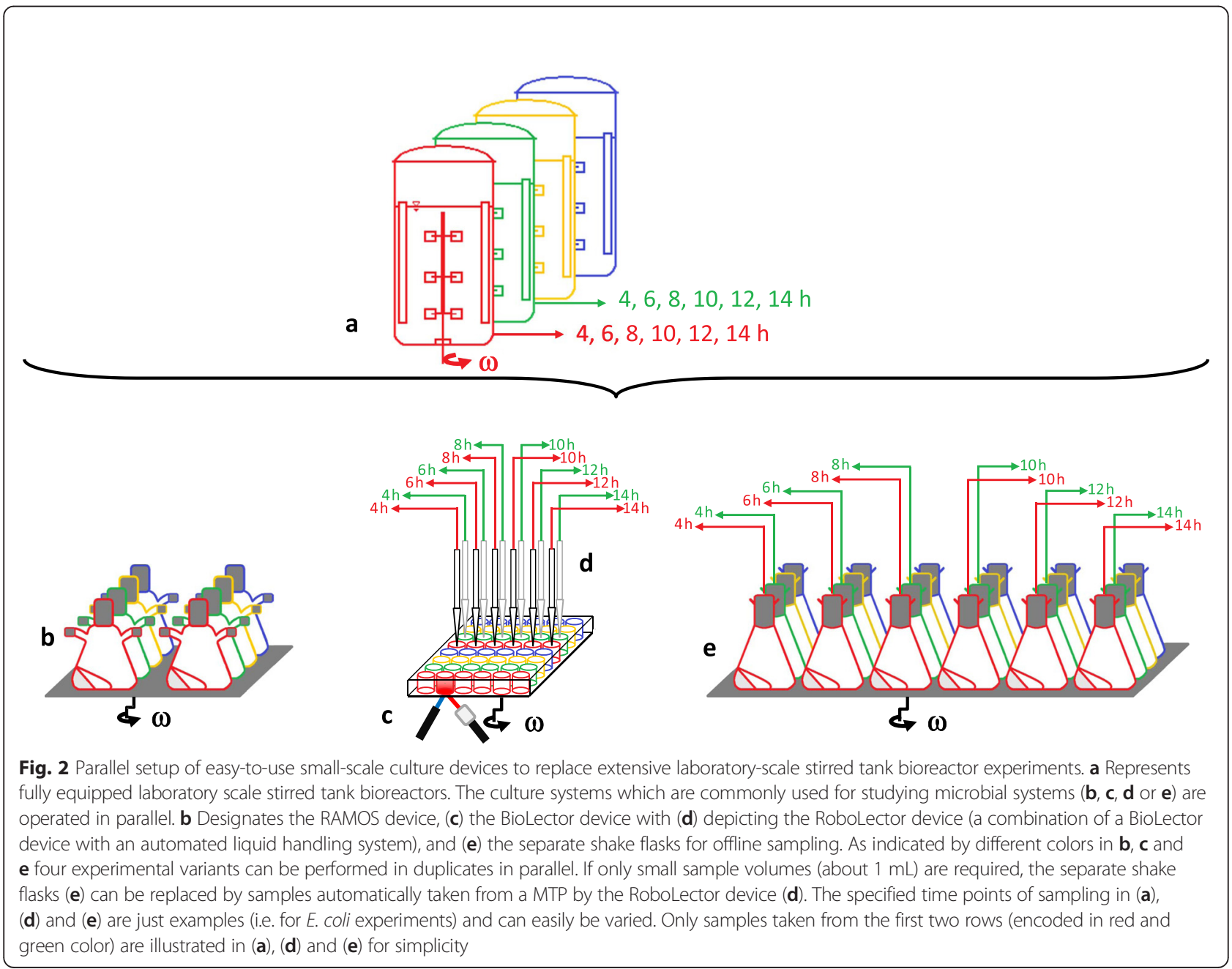


distributed among the different bioreactors resulting in "one experiment".

Many studies focused on various process-parameters (oxygen transfer, power input, mixing time etc.) as critical scaling parameter and were recently summarized by Marques et al. [23]. Fermentation processes can be successfully scaled-up from shake flasks to laboratory-scale stirred tank bioreactor [24-26] as well as from MTPs to laboratory-scale stirred tank bioreactor [21, 27-29] and even further to pilot-scale stirred tank bioreactor [26, 29]. Scale-up is, therefore, not in the focus of this study. The herein presented approach focusses on the oxygen transfer rate (OTR), which is a key parameter for the evaluation of a cultivation progress. As demonstrated by Anderlei et al. [13], many physiological states of cells - e.g. diauxic growth or limitation by oxygen or by primary as well as secondary substrates - can be identified by the progression of the OTR during a cultivation.

We show that the step of strain selection and strain evaluation as well as the initial process development (step 4 and 5 in Fig. 1) in a laboratory-scale stirred tank bioreactor can be replaced by the new experimental approach (Fig. 2). For the new approach, already existing culture devices can be used. Although for final process optimization experiments (step 6 in Fig. 1) laboratory scale stirred tank bioreactors are still necessary, the total number of stirred tank bioreactor experiments can significantly be reduced.

\section{Results and discussion}

Determination of the medium-specific maximum oxygen transfer capacity in 48-well microtiter plates and $250 \mathrm{~mL}$ shake flasks

To obtain equal cultivation conditions in microtiter plates (48-well Flowerplate) and $250 \mathrm{~mL}$ shake flasks, it is important to guarantee equal maximum oxygen transfer capacity $\left(\mathrm{OTR}_{\max }\right)$ values. This fact is especially important in case of oxygen limitation which will be examined in a later section.

Using Escherichia coli BL21 EcFbFP in modified

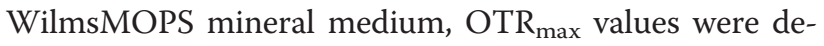
termined as described in the Methods section (data shown in Additional file 1: Figure S1, Additional file 2: Figure S2 and Additional file 3: Figure S3). Figure 3 illustrates the dependency of the $\mathrm{OTR}_{\max }$ value on the filling volume in a 48-well Flowerplate and a $250 \mathrm{~mL}$ nonbaffled shake flask. For MTP cultivations, different shaking frequencies are displayed, also. In general, a decreasing filling volume results in an increasing $\mathrm{OTR}_{\max }$ value. Additionally, an increase in the $\mathrm{OTR}_{\max }$ value can be obtained by increasing the shaking frequency. These findings agree with the results published earlier $[8,12]$. The small shaking diameter $(3 \mathrm{~mm}$ ) used for deep well MTP cultivations allows at very high shaking frequencies (n) much higher

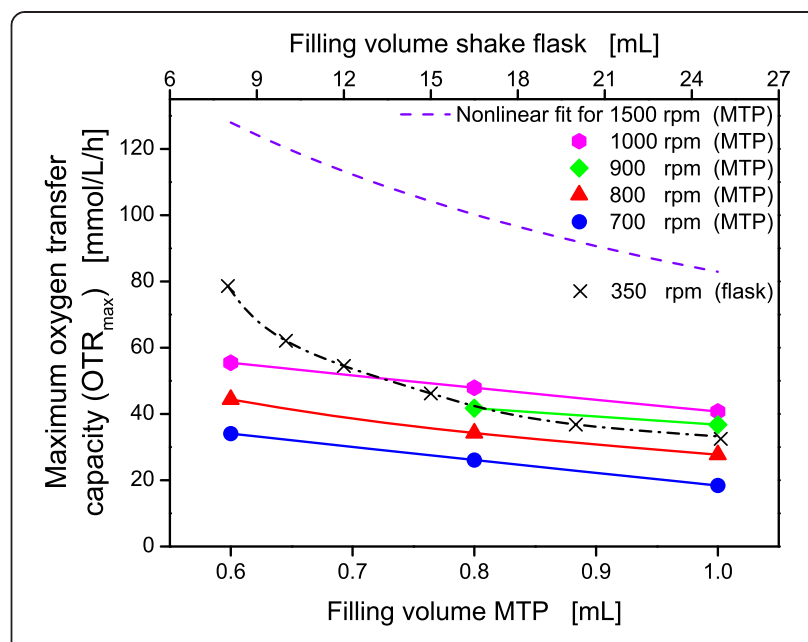

Fig. 3 Dependencies of the OTR $\max$ for E. coli BL21 EcFbbF in shake flasks and 48-well Flowerplate. Culture conditions in RAMOS: WilmsMOPS medium supplemented with $20 \mathrm{~g} / \mathrm{L}$ glucose, buffered with $0.2 \mathrm{M}$ MOPS, $250 \mathrm{~mL}$ RAMOS shake flasks, $V_{\mathrm{L}}=8-25 \mathrm{~mL}$, $\mathrm{n}=350 \mathrm{rpm}, \mathrm{d}_{0}=50 \mathrm{~mm}, \mathrm{~T}=37^{\circ} \mathrm{C}$. Culture conditions for microRAMOS: WilmsMOPS medium supplemented with $20 \mathrm{~g} / \mathrm{L}$ glucose, buffered with $0.2 \mathrm{M} \mathrm{MOPS,} \mathrm{48-} \mathrm{well} \mathrm{Flowerplate,} V_{L}=0.6-1 \mathrm{~mL}, \mathrm{n}=700$ $1000 \mathrm{rpm}, \mathrm{d}_{0}=3 \mathrm{~mm}, \mathrm{~T}=37^{\circ} \mathrm{C}$. To estimate the OTR $\max$ values at a shaking frequency of $1500 \mathrm{rpm}$, a nonlinear fit, incorporating all data shown for the microtiter plate in this figure, was calculated, thereby resulting in the following equation: OTR $\max =1.65 \cdot 10^{-4} \cdot V_{L}^{-0.85} \cdot n^{1.795}$

OTR $_{\max }$ and $\mathrm{k}_{\mathrm{L}}$ a values than compared to those of shake flasks which are shaken (maximum at $350 \mathrm{rpm}$ ) at a larger shaking diameter $(50 \mathrm{~mm})$. As recently reported by Bareither and Pollard, $\mathrm{k}_{\mathrm{L}}$ a values of up to $800 \mathrm{~h}^{-1}$ resulting in OTR values of up to $200 \mathrm{mmol} / \mathrm{L} / \mathrm{h}$ can be reached applying a small shaking diameter of $3 \mathrm{~mm}$ paired with a high shaking frequency of $1400 \mathrm{rpm}$ in square-shaped 96deep-well plates [1]. The herein presented $\mathrm{OTR}_{\max }$ values for a 48-well Flowerplate (MTP) were obtained by conducting measurements in a MicroRAMOS device as described by Kensy et al. [16] and were restricted to a maximum shaking frequency of $1000 \mathrm{rpm}$. In order to determine the necessary shaking frequency and filling volume for non-oxygen limited conditions a non-linear regression was made. The regression model incorporates all $\mathrm{OTR}_{\max }$ values obtained in MTPs for different filling volumes $\left(\mathrm{V}_{\mathrm{L}}\right)$ and shaking frequencies $(\mathrm{n})$ ranging from $0.6 \mathrm{~mL}$ to $1 \mathrm{~mL}$ and $700 \mathrm{rpm}$ to $1000 \mathrm{rpm}$, respectively. The resulting equation for calculating the $\mathrm{OTR}_{\max }$ value is:

$$
\mathrm{OTR}_{\max }=1.65 \cdot 10^{-4} \cdot \mathrm{V}_{\mathrm{L}}^{-0.85}[\mathrm{~mL}] \cdot \mathrm{n}^{1.795}\left[\mathrm{~min}^{-1}\right]
$$

This equation is specific only for the applied WilmsMOPS mineral medium because a different medium would lead to a different oxygen solubility and different $\mathrm{OTR}_{\max }$ 
values. With this equation, OTR $\mathrm{Omax}_{\operatorname{mal}}$ values specific for the cultivation of E. coli BL21 EcFbFP in WilmsMOPS mineral medium could be calculated for any combination of shaking frequency and filling volume. As an example, a curve for $1500 \mathrm{rpm}$ and varying filling volumes $(0.6-$ $1 \mathrm{~mL}$ ) is shown in Fig. 3.

\section{Non-oxygen-limited cultivations}

Due to the fact that the E. coli BSLA EcFbFP culture is still limited applying $8 \mathrm{~mL}$ filling volume, $350 \mathrm{rpm}$ shaking frequency and $20 \mathrm{~g} / \mathrm{L}$ glucose (Additional file 1: Figure S1), there are four factors that can be modified to meet the requirements for non-oxygen-limited condition: (1) decrease the filling volume; (2) increase the shaking frequency; (3) aeration with oxygen enriched air; (4) decrease the sugar concentration. Option 1-3 are not favorable due to practical reasons. A further decrease of the filling volume would lead to unforeseeable evaporation problems. A further increase of the shaking frequency is restricted by the technical design of the orbital shaker. Aeration with oxygen enriched air is expensive, laborious and working with pure oxygen is risky. Therefore, the glucose concentration was decreased and the $\mathrm{OTR}_{\max }$ values for WilmsMOPS mineral medium supplemented with $10 \mathrm{~g} / \mathrm{L}$ glucose were determined (see Additional file 3: Figure S3). For $8 \mathrm{~mL}$ and $350 \mathrm{rpm}$ no oxygen limitation could be observed. This result indicates that an OTR $\mathrm{max}_{\max }$ value of somewhat more than $70 \mathrm{mmol} /$ $\mathrm{L} / \mathrm{h}$ has to be guaranteed in order to avoid oxygen limitation in WilmsMOPS mineral medium supplemented with $10 \mathrm{~g} / \mathrm{L}$ glucose at $37{ }^{\circ} \mathrm{C}$. This prior information about oxygen requirement of the investigated strains is essential and should be generated in primary and secondary strain screening (steps 2 and 3 in Fig. 1).

After the mastermix was prepared, it was distributed among RAMOS flasks ( $8 \mathrm{~mL}$ each), separate shake flasks $(8 \mathrm{~mL}$ each) and 6 wells of the Flowerplate $(1 \mathrm{~mL}$ per well). By using one mastermix for all three small-scale culture systems, errors due to medium preparation and inoculation could be avoided. Non-oxygen limited conditions for cultivation with $10 \mathrm{~g} / \mathrm{L}$ glucose can be expected for shake flask cultivation by applying the following parameters: $50 \mathrm{~mm}$ shaking diameter, $350 \mathrm{rpm}$ shaking frequency and $8 \mathrm{~mL}$ filling volume resulting in a measured OTR value of $71 \mathrm{mmol} / \mathrm{L} / \mathrm{h}$. For the Flowerplate (MTP) the suggested cultivation parameters are: $3 \mathrm{~mm}$ shaking diameter, $1500 \mathrm{rpm}$ shaking frequency and $1 \mathrm{~mL}$ filling volume resulting in a calculated OTR value of $82.9 \mathrm{mmol} / \mathrm{L} / \mathrm{h}$.

Figure $4 \mathrm{a}, \mathrm{c}$ and e show the results of the non-oxygenlimited cultivation of E. coli BL21 EcFbFP. The comparison of the oxygen transfer rate (OTR) and the dissolved oxygen tension (DOT) measured by RAMOS and BioLector devices, respectively, is illustrated in Fig. 4a. The
OTR curve demonstrates the characteristic evolution of oxygen consumption over time for a non-oxygen limited E. coli cultivation, comparable to previous results [19]. The OTR curve shows no plateau, which indicates that the cultivation was not subject to oxygen limitation at any time. The DOT curve is a mirror image of the OTR curve and displays no oxygen limitation throughout the whole cultivation (DOT $\geq 40 \%$ ). Therefore, the estimations for non-oxygen limited conditions made in the previous section were proven to be correct. The OTR curve exponentially increases (DOT curve drops) until complete consumption of the substrate after $8 \mathrm{~h}$. As described in the Methods section, the $\mathrm{k}_{\mathrm{L}} \mathrm{a}$ value was calculated by Equation 2 from the DOT allowing the calculation of $\mathrm{OTR}_{\text {calc }}$ values in the MTPs. As shown in Fig. $4 \mathrm{a}$, the progression of OTR $\mathrm{Calc}_{\mathrm{c}}$ in the MTPs and the measured OTR in shake flasks almost concur. However, due to the restriction of the number of measuring points over time for the OTR in shake flasks, the maximum OTR value was not detected. Since the OTR is measured every $30 \mathrm{~min}$ and the increase in DOT and the decrease in OTR are identical, most probably the actual OTR peak was missed.

Figure $4 \mathrm{c}$ shows that biomass $\left(\mathrm{OD}_{600}\right)$ is produced during the exponential growth phase while the glucose is consumed and small amounts of acetate are produced simultaneously (Fig. 4e) [30]. The end of exponential biomass production is observed at the same time point at which the OTR decreases and the DOT rises $(8 \mathrm{~h})$ and was also detected non-invasively, online using the scattered light (S.L., Fig. 4c) measurement installed in the BioLector device. The small increase in the value for S.L. intensity after the OTR curve drops down is most likely due to morphological changes as described in previous publications [31-33].

Between 8 and $10.5 \mathrm{~h}$, the acetate is gradually consumed, leading to the shoulder in the OTR curve. Production and consumption of acetate was also monitored by the $\mathrm{pH}$-value (Fig. 4e). Starting at a value of 7.5 the $\mathrm{pH}$-value steadily decreases to a minimum of 7.0 within $8 \mathrm{~h}$ and rises again to a final value of 7.1 as the acetate is consumed. In Fig. 4e, the fluorescence tag signal (EcFbFP) is also plotted as a function over time. The fluorescence activity is relatively low as this culture is not induced by IPTG.

\section{Oxygen-limited E.coli cultivations}

Many commonly applied screening conditions lead to oxygen limitation due to unfavorable cultivation parameters. High filling volumes and low shaking frequencies can cause oxygen limitation and, hence, trigger unfavorable effects like formation of products of anaerobic metabolism, excessive $\mathrm{pH}$-shifts and growth inhibition $[34,35]$. Characterizing the influence of oxygen limitation 


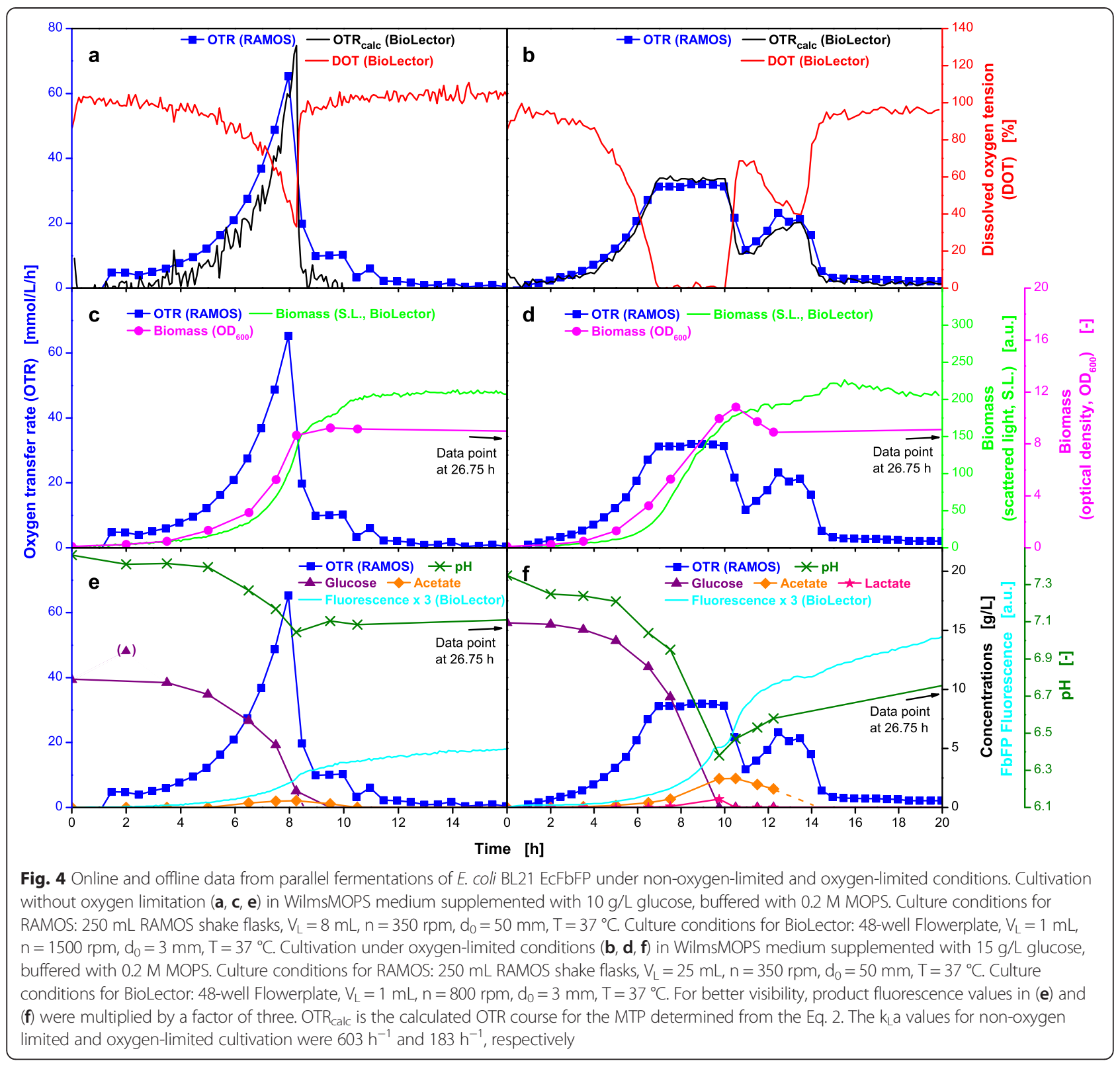

is also a relevant subject for scale-up [36], whereas in this case oxygen limitations due to inhomogenities in large scale cannot be compared with the herein triggered oxygen limitations. To simulate oxygen-limited conditions, cultivation parameters were chosen that lead to an $\mathrm{OTR}_{\max }$ value of about $30 \mathrm{mmol} / \mathrm{L} / \mathrm{h}$. For the shake flask cultivation a filling volume of $25 \mathrm{~mL}$ at $350 \mathrm{rpm}$ and for the MTP a filling volume of $1 \mathrm{~mL}$ at $800 \mathrm{rpm}$ resulted in almost identical OTR $\mathrm{max}_{\max }$ values (Fig. 3).

Figure $4 \mathrm{~b}$ shows the curves of OTR and DOT for the oxygen-limited cultivation of the same $E$. coli strain discussed in Fig. 4a, c and e. The DOT curve is an inverse of the OTR curve (shake flask) which concurs very well with the OTR calc $(\mathrm{MTP})$. This observation demonstrates that the RAMOS and BioLector devices were operated at conditions providing the same oxygen supply. Combining both measurement techniques, therefore, reveals a great potential. Obviously equivalent $\mathrm{OTR}_{\max }$ values could successfully be adjusted in shake flasks and MTPs. The exponential growth lasts until $7 \mathrm{~h}$ and is then immediately interrupted by the oxygen limitation at an OTR value of $32 \mathrm{mmol} / \mathrm{L} / \mathrm{h}$. The plateau of the OTR curve lasts for $3 \mathrm{~h}$ while the glucose is consumed before it decreases to $11.8 \mathrm{mmol} / \mathrm{L} / \mathrm{h}$ and rises again to a maximum of $24 \mathrm{mmol} /$ $\mathrm{L} / \mathrm{h}$ during consumption of overflow metabolites (Fig. 4f) [37]. It finally decreases to about $0 \mathrm{mmol} / \mathrm{L} / \mathrm{h}$ after $15 \mathrm{~h}$.

The biomass production under oxygen-limited conditions is displayed in Fig. 4d. The $\mathrm{OD}_{600}$ increases exponentially 
until $7 \mathrm{~h}$ followed by a linear progression until $10.5 \mathrm{~h}$. After the maximum $\mathrm{OD}_{600}$ of 10.8 is reached, a small drop occurs and afterwards remains constant at a level of about 9. The same trend can also be recognized for online monitored S.L. intensity using the BioLector device (Fig. 4d). The further increase in S.L. after carbon source depletion has already been discussed for non-oxygen limited conditions in the previous section.

Due to the high concentration of overflow metabolites, a diauxic growth can be observed and the OTR shows two peaks (Fig. 4b, d and f) [38]. The formation of $3.1 \mathrm{~g} / \mathrm{L}$ of total organic acids explains the reduction in the $\mathrm{pH}$ value to 6.4. The last offline sample was taken after $26.75 \mathrm{~h}$ and no acetate could be detected. Given the fact that the second OTR peak represents the uptake and consumption of acetate, the dashed line indicates the real progression of the acetate curve and the time point of depletion, which occurs with drop down of the OTR curve after $14 \mathrm{~h}$. For this cultivation, carbon balances have been calculated and could be closed to $96 \%$. As for non-limited conditions only a low fluorescence signal indicative for product formation is detected due to missing induction.

\section{Diauxic growth and comparability of offline samples from MTP and shake flask}

To challenge our new experimental approach, growth under diauxic conditions was examined using $20 \mathrm{~g} / \mathrm{L}$ glucose and $1.5 \mathrm{~g} / \mathrm{L}$ sorbitol as carbon sources [38, 39]. Diauxic growth on several carbon sources results in more distinct peaks and "structure" in the OTR and DOT curves, making comparison of data from shake flasks and microtiter plates more sensitive. As for the previously described experiments, RAMOS, RoboLector and separate shake flasks were run in parallel. In this trial, additional samples for offline analysis were taken directly from the MTP with a RoboLector [17]. Overflow metabolism is intentionally triggered by cultivation conditions that lead to oxygen limitation. Cultivation parameters were chosen using the data from Fig. 3 to obtain equal OTR $\max$ values (about $55 \mathrm{mmol} / \mathrm{L} / \mathrm{h}$ ) causing a slight oxygen limitation. Measuring points closest to the desired $\mathrm{OTR}_{\max }$ value were obtained at $12 \mathrm{~mL}$ and $350 \mathrm{rpm}$ in shake flasks and $0.6 \mathrm{~mL}$ and $1000 \mathrm{rpm}$ in MTPs. The experiment was performed at $30{ }^{\circ} \mathrm{C}$ to slow down the metabolic activity of the $E$. coli strain in order to ensure observability of details of the metabolic behavior. The expected OTR $\mathrm{max}_{\max }$ value will be slightly higher than the value displayed in Fig. 3 because a lower temperature $\left(30{ }^{\circ} \mathrm{C}\right.$ instead of $\left.37{ }^{\circ} \mathrm{C}\right)$ results in an increased solubility for oxygen and, therefore, a higher OTR $_{\max }$. This is partially compensated by lower diffusion coefficients as shown by Krahe et al. [40].

Figure $5 \mathrm{a}$ shows the courses of the OTR and DOT curves of the RAMOS and RoboLector devices, respectively.
Additionally, the OTR calc from the DOT values of the MTP is displayed. During the first $17 \mathrm{~h}$, the DOT and $\mathrm{OTR}_{\text {calc }}$ (MTP) as well as measured OTR (shake flask) match. Due to a slightly higher biomass in the MTP $\left(\mathrm{OD}_{600}\right.$, Fig. $\left.5 \mathrm{~b}\right)$ the consumption of overflow metabolites occurs sooner (starting at $17 \mathrm{~h}$ ) in the MTP cultivation than compared to shake flask cultivation. The $\mathrm{OD}_{600}$ values from shake flasks and MTPs were measured, and the results are displayed as circles in Fig. 5b. The line through the circles represents the average of both samples. Even though the cultivation vessels are completely different, the values for $\mathrm{OD}_{600}$ agree very well. This indicates that all growth-relevant parameters were identical in shake flasks and MTPs under the applied operating conditions.

Within the first $16 \mathrm{~h}$ of incubation, $2.1 \mathrm{~g} / \mathrm{L}$ acetate is produced and glucose is completely consumed which is indicated by the first drop in the OTR curve also resulting in a decreasing $\mathrm{pH}$-value (Fig. 5c). After this decrease, consumption and depletion of the $1.5 \mathrm{~g} / \mathrm{L}$ sorbitol takes place within only $1 \mathrm{~h}(16.5-17.5 \mathrm{~h})$. Subsequently and, in part, simultaneously to the uptake of sorbitol, the $\mathrm{pH}$-value rises because the overflow metabolite acetate is consumed. This observation explains the rise and fall of the OTR curve between $17.5 \mathrm{~h}$ and $20 \mathrm{~h}$ representing the third peak. Therefore, this culture has to be regarded as a triauxic system [38], although only two different carbon sources have been added at the beginning. Calculating the carbon balance of this microbial system showed that the balance could be closed to a value up to $98 \%$. Results in Fig. $5 \mathrm{~b}$ and c demonstrate how $\mathrm{OD}_{600}$, the concentration of glucose, sorbitol, acetate and the $\mathrm{pH}$-value concur nicely in the samples from the shake flasks and MTPs. All offline measurements of the different parameters agree very well with each other.

\section{E. coli in mineral auto-induction medium}

In a defined mineral auto-induction medium experiment, E. coli BL21 BSLA was cultured in parallel in the RAMOS and BioLector devices. The WilmsMOPS mineral auto-induction medium was supplemented with $0.5 \mathrm{~g} / \mathrm{L}$ glucose as substrate for the initial growth and to repress the lac operon [41]. Moreover, the medium contained $2 \mathrm{~g} / \mathrm{L}$ lactose to induce the lac operon (after glucose depletion) and $5 \mathrm{~g} / \mathrm{L}$ glycerol as additional carbon source. To avoid negative effects of oxygen limitation, conditions were chosen at which the oxygen supply is sufficient in the RAMOS and BioLector devices $\left(\mathrm{OTR}_{\max } \geq\right.$ $60 \mathrm{mmol} / \mathrm{L} / \mathrm{h}$ ).

As shown in Fig. 6a, identically cultivation conditions in the RAMOS and BioLector devices could be established. An initial increase in the OTR curve occurs due to growth on the preferred carbon source glucose. After $3 \mathrm{~h}$, the depletion of glucose (Fig. 6c) leads to a reproducible small 


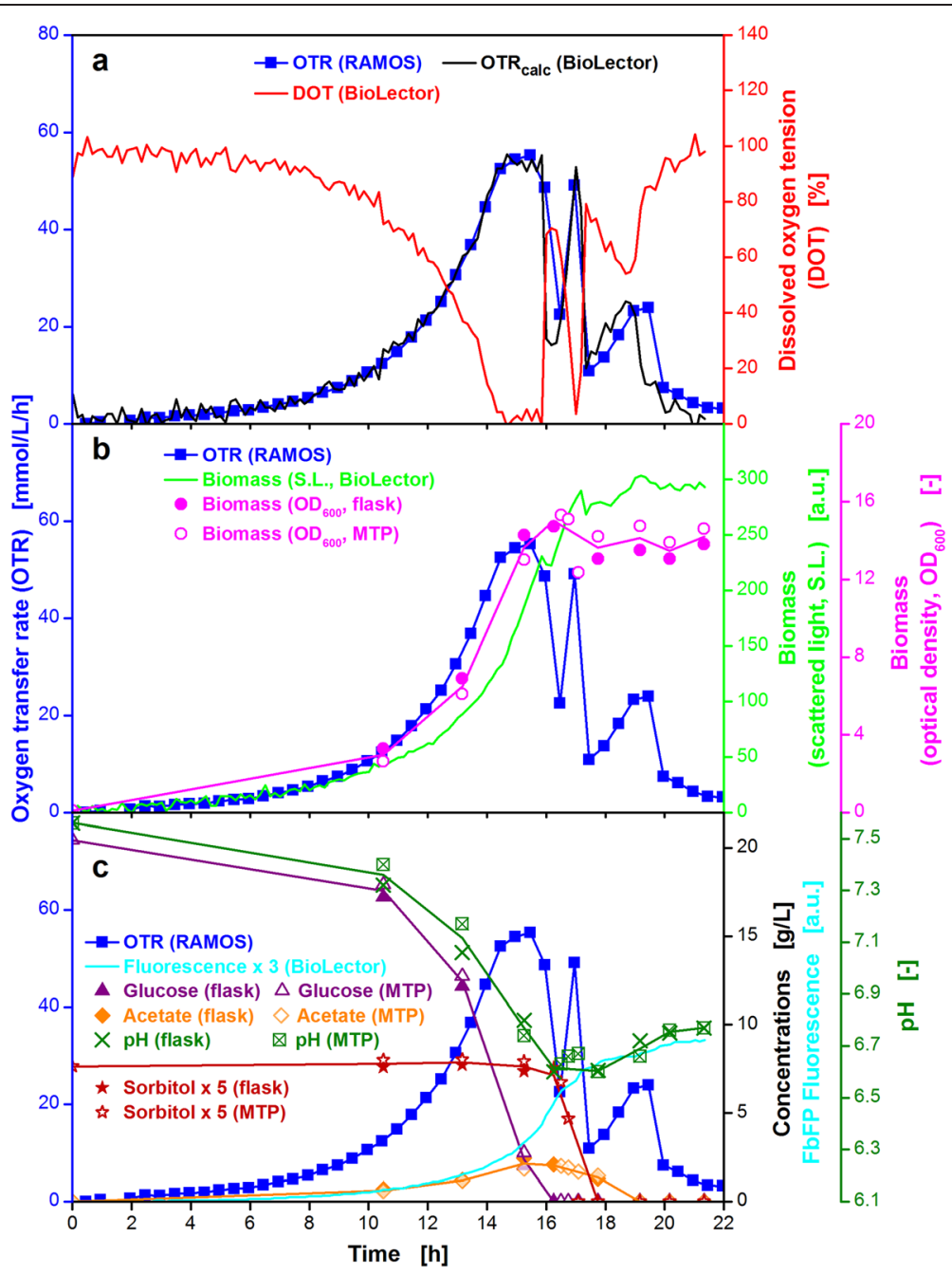

Fig. 5 Online and offline data from parallel fermentations of E. coli BL21 EcFbFP under diauxic conditions. Cultivation in WilmsMOPS mineral medium supplemented with $20 \mathrm{~g} / \mathrm{L}$ glucose and $1.5 \mathrm{~g} / \mathrm{L}$ sorbitol, buffered with $0.2 \mathrm{M}$ MOPS. Culture conditions for RAMOS: $250 \mathrm{~mL}$ RAMOS shake flasks, $V_{L}=12 \mathrm{~mL}, \mathrm{n}=350 \mathrm{rpm}, \mathrm{d}_{0}=50 \mathrm{~mm}, \mathrm{~T}=30^{\circ} \mathrm{C}$. Culture conditions for RoboLector: 48-well Flowerplate, $V_{\mathrm{L}}=0.6 \mathrm{~mL}, \mathrm{n}=1000 \mathrm{rpm}, \mathrm{d}_{0}=3 \mathrm{~mm}$, $\mathrm{T}=30^{\circ} \mathrm{C}$. Lines shown in (b) and (c) for $\mathrm{OD}_{600}$, glucose, sorbitol, acetate and $\mathrm{pH}$ represent the averages of the offline data obtained from $250 \mathrm{~mL}$ shake flasks and 48-well microtiter plate at the same time points. For better visibility, the sorbitol concentrations were multiplied by a factor of five and the product fluorescence values by a factor of three. The $k_{\llcorner}$a value used for calculation of the OTR calc course was (a) $276 \mathrm{~h}^{-1}$

drop in the OTR curve. The further increase to a second OTR peak at $10 \mathrm{mmol} / \mathrm{L} / \mathrm{h}$ after $5 \mathrm{~h}$ is due to growth on glycerol. Afterwards, the OTR curve slightly decreases (5$12 \mathrm{~h}$ ) due to the metabolic burden imposed by the production of the tagged fluorescent protein (FbFP-BLSA) during lactose consumption [42, 43]. After lactose depletion, growth on the residual glycerol leads to an increase in the OTR curve resulting in a third peak at $21 \mathrm{mmol} / \mathrm{L} / \mathrm{h}$. The end of the cultivation (after depletion of all carbon sources) is indicated by the sharp drop in the OTR curve. Similar OTR curves were described by Kunze et al. and Rahmen et al. on complex auto-induction medium $[44,45]$. The progression of the OTR curve is well mirrored by the progression of the DOT curve. The trend of the calculated OTR in MTPs $\left(\mathrm{OTR}_{\text {calc }}\right)$ agrees with the measured OTR curve in shake flasks. However, this example shows the limits of calculating OTR values from DOT values (with the aid of the respective $\mathrm{k}_{\mathrm{L}} \mathrm{a}$ values). The accuracy of the OTR directly measured by the RAMOS technique is clearly superior.

The biomass increased exponentially until $6 \mathrm{~h}$ of cultivation, followed by a further linear increase (S.L., Fig. 6b). The time point of the shift from exponential to linear growth roughly correlates with the second peak of the OTR curve. The duration of the linear growth (5-12 h) concurs with the expression phase of the fluorescent tagged product (Fig. 6c). With the final increase in the OTR values, additional biomass is produced. The $\mathrm{OD}_{600}$ 


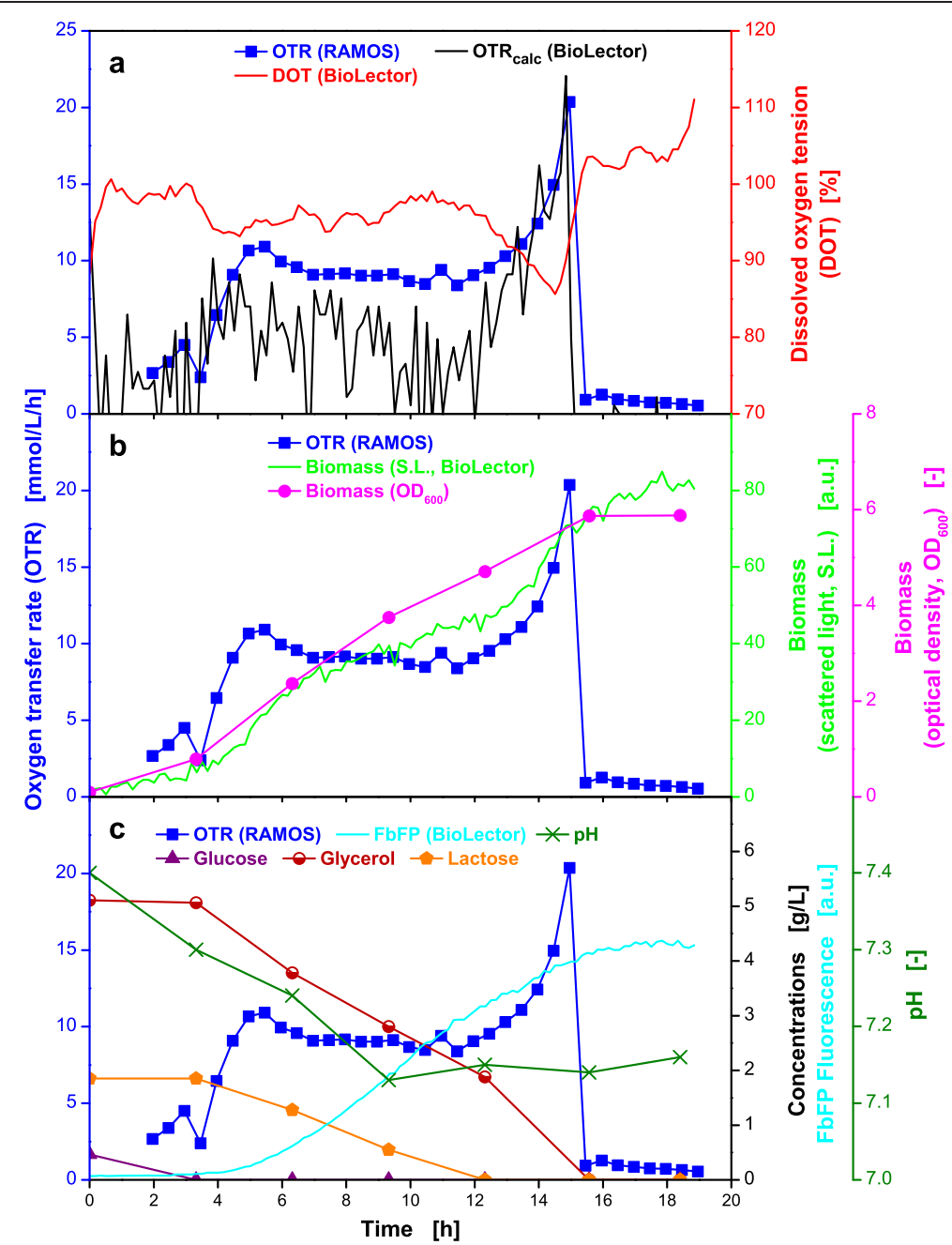

Fig. 6 Online and offline data from parallel fermentations of E. coli BL21 BSLA in auto-induction medium. Cultivation in WilmsMOPS mineral autoinduction medium containing $0.5 \mathrm{~g} / \mathrm{L}$ glucose, $2 \mathrm{~g} / \mathrm{L}$ lactose and $5 \mathrm{~g} / \mathrm{L}$ glycerol, buffered with $0.2 \mathrm{M} \mathrm{MOPS} \mathrm{(a-c).} \mathrm{Culture} \mathrm{conditions} \mathrm{for} \mathrm{RAMOS:}$ $250 \mathrm{~mL}$ RAMOS shake flasks, $V_{L}=10 \mathrm{~mL}, \mathrm{n}=350 \mathrm{rpm}, \mathrm{d}_{0}=50 \mathrm{~mm}, \mathrm{~T}=37^{\circ} \mathrm{C}$. Culture conditions for BioLector: 48-well Flowerplate, $V_{\mathrm{L}}=1 \mathrm{~mL}$, $\mathrm{n}=1500 \mathrm{rpm}, \mathrm{d}_{0}=3 \mathrm{~mm}, \mathrm{~T}=37^{\circ} \mathrm{C}$. The $\mathrm{k}_{\mathrm{L}}$ a value used for calculation of the OTR calc course was $587 \mathrm{~h}^{-1}$

curve points to linear growth throughout the whole cultivation which is most likely attributed to the few sampling points only every two hours. This affirms the value of online monitoring and corresponding signals.

The $\mathrm{pH}$-value changes only moderately, because oxygen limitation and formation of products of anaerobic metabolism could be avoided and, thus, no acidic byproducts were formed [37]. The fluorescence signal rises to a value of about 4.5 a.u.

\section{Gluconobacter oxydans cultivations on complex medium}

The acetic acid bacterium Gluconobacter oxydans DSM 3504 was additionally tested to verify the proposed new experimental approach for other microorganisms than $E$. coli. Figure 7 displays the comparison of an unbuffered (Fig. 7a, c and xe) and PIPPS buffered (Fig. 7b, d and f) cultivations of the strain G. oxydans 3504 under non- oxygen limited conditions. Both experiments were performed simultaneously using a RAMOS and BioLector device. In both cases the OTR curve increases exponentially until it reaches the maximum after $5 \mathrm{~h}$. The first difference between unbuffered and buffered cultivation is visible by the maximum OTR values reached. Under buffered conditions a higher OTR maximum $(43.4 \mathrm{mmol} /$ $\mathrm{L} / \mathrm{h}$, Fig. 7b) is observed as compared to unbuffered conditions $(34.8 \mathrm{mmol} / \mathrm{L} / \mathrm{h}$, Fig. 7a). In both cases this behavior is mirror imaged by the DOT value, which drops to a minimum of $69 \%$ and $53 \%$ for the unbuffered and buffered cultivation, respectively. Further on, the OTR steadily decreases for the unbuffered cultivation while for the buffered cultivation a second OTR maximum of $20.8 \mathrm{mmol} /$ $\mathrm{L} / \mathrm{h}$ is reached after $11 \mathrm{~h}$. Both progressions of the OTR curves are mirrored by the DOT throughout the whole cultivation time. Additionally, the $\mathrm{OTR}_{\text {calc }}$ courses for 


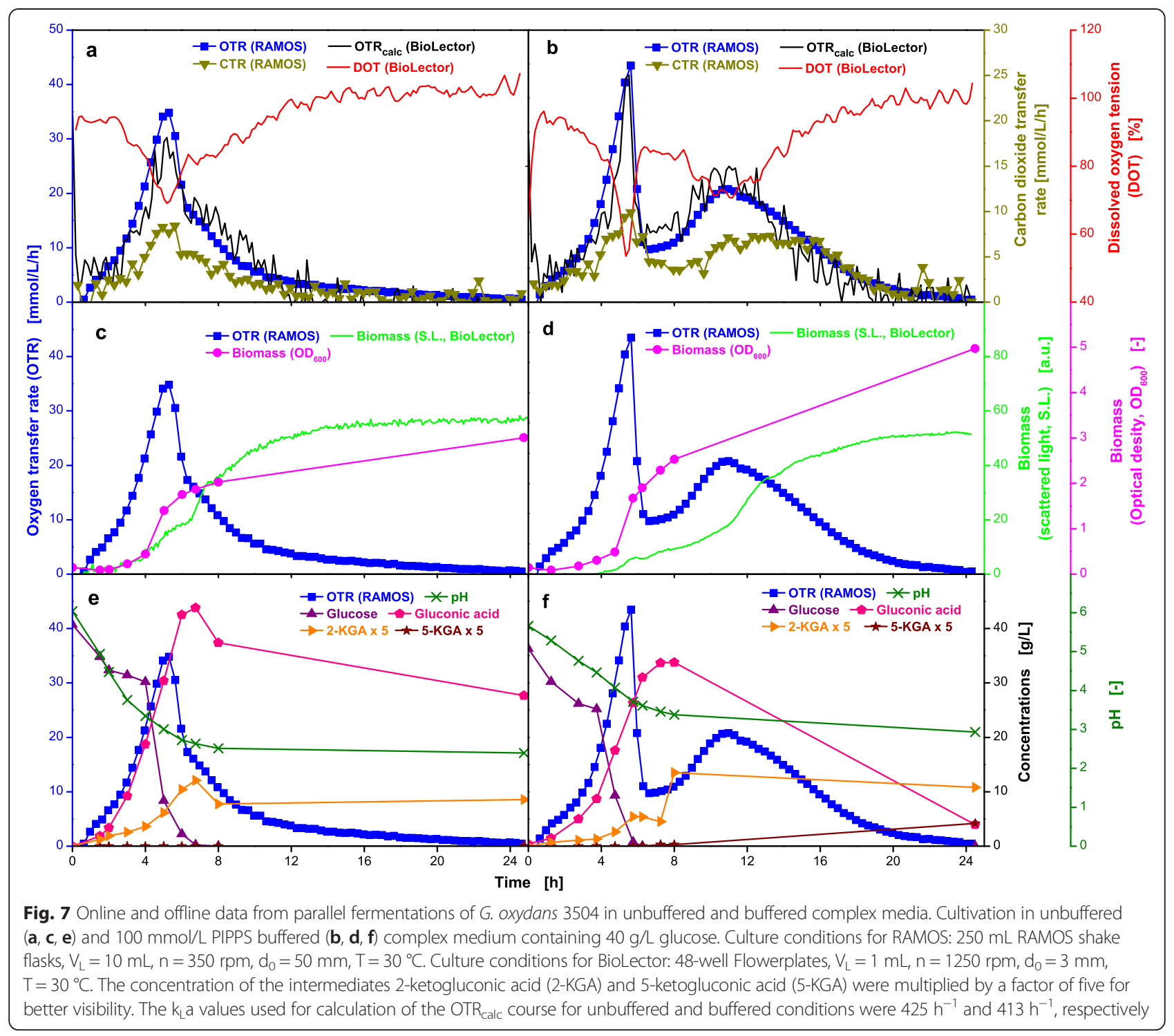

MTP cultivations, which were calculated from the measured DOT curves, are in accordance with the measured OTR courses of the shake flasks.

During the cultivation, the CTR is lower than the OTR $(R Q<1)$, suggesting that not all consumed oxygen was directed into biomass production and that an additional oxidative pathway is active. Taking the curves of the $\mathrm{OD}_{600}$ and scattered light measurement (Fig. 7c, d) as well as the progression of the concentration of glucose and its respective oxidation products (gluconic acid, 2-ketogluconic acid and 5-ketogluconic acid, Fig. 7e, f) into account, it becomes clear that the membrane bound oxidases are active, which require a portion of the consumed oxygen [46]. Gluconic acid was continuously formed until glucose was almost depleted and subsequently ketogluconic acids were formed. The formation of 5-ketogluconic acid can only be observed for the
PIPPS buffered cultivation because the $\mathrm{pH}$-value remains above 3, allowing glucose dehydrogenase activity [47].

Differences in $\mathrm{OD}_{600}$ and S.L. become obvious in Fig. $7 \mathrm{c}, \mathrm{d}$. While for unbuffered conditions the $\mathrm{OD}_{600}$ and S.L. curves partly concur, they show different courses for the buffered cultivation. This observation may be caused by an altered morphology of the cells as described by Kunze et al. [48]. As recently published, soluble buffers significantly increase the osmotic stress and therefore influence the oxygen consumption of G. oxydans 3504 [49]. Hence, it is highly conceivable that the osmotic pressure also influences the cell morphology.

Two different experiments (unbuffered and $100 \mathrm{mmol} / \mathrm{L}$ PIPPS) were simultaneously preformed resulting in a data density acquired so far only from cultivations in fully equipped laboratory-scale stirred tank bioreactors. Additionally, very interesting findings could be made by 
monitoring the S.L. intensity and $\mathrm{OD}_{600}$ values during the cultivations. Two additional cultivations were performed simultaneously as well, with different buffer concentrations (data not shown) which adds up to four different experimental variants at the same time as illustrated in Fig. 2. As an important advantage, all four experimental variants were performed in duplicate (in RAMOS) and in triplicate (in BioLector) to verify the repeatability of the obtained results.

\section{Oxygen-limited cultivation of the yeast Kluyveromyces lactis on complex medium}

In order to prove the applicability of the proposed new experimental approach for yeast cultivations, the genetically modified strain Kluyveromyces lactis Cel5A was investigated. In addition to oxygen limitation, a high substrate concentration ( $30 \mathrm{~g} / \mathrm{L}$ galactose) was applied to cause overflow metabolism.
The curves for OTR and DOT plotted in Fig. 8a show a steep increase or decrease, respectively, which is caused by an exponential growth phase until $11 \mathrm{~h}$. During 12 and $20 \mathrm{~h}$, the yeast grows linearly due to the oxygen-limited culture conditions. Both signals, OTR and DOT, from shake flask and microtiter plate are in agreement with respect to start and end of oxygen limitation. The OTR calc calculated from the DOT values of the MTP (Eq. 2) concurs nicely with the measured OTR in shake flasks, suggesting equivalent environmental conditions for the microorganism in shake flasks and MTPs. The hypothesis of changing from exponential to linear growth can be verified by the evolution of the biomass curves (S.L. intensity, $\mathrm{OD}_{600}$ and cell dry weight) plotted in Fig. 8b. All three biomass values (online and offline) show the same course, which indicates that an influence of the cell morphology on the scattered light intensity for $K$. lactis can be excluded under these

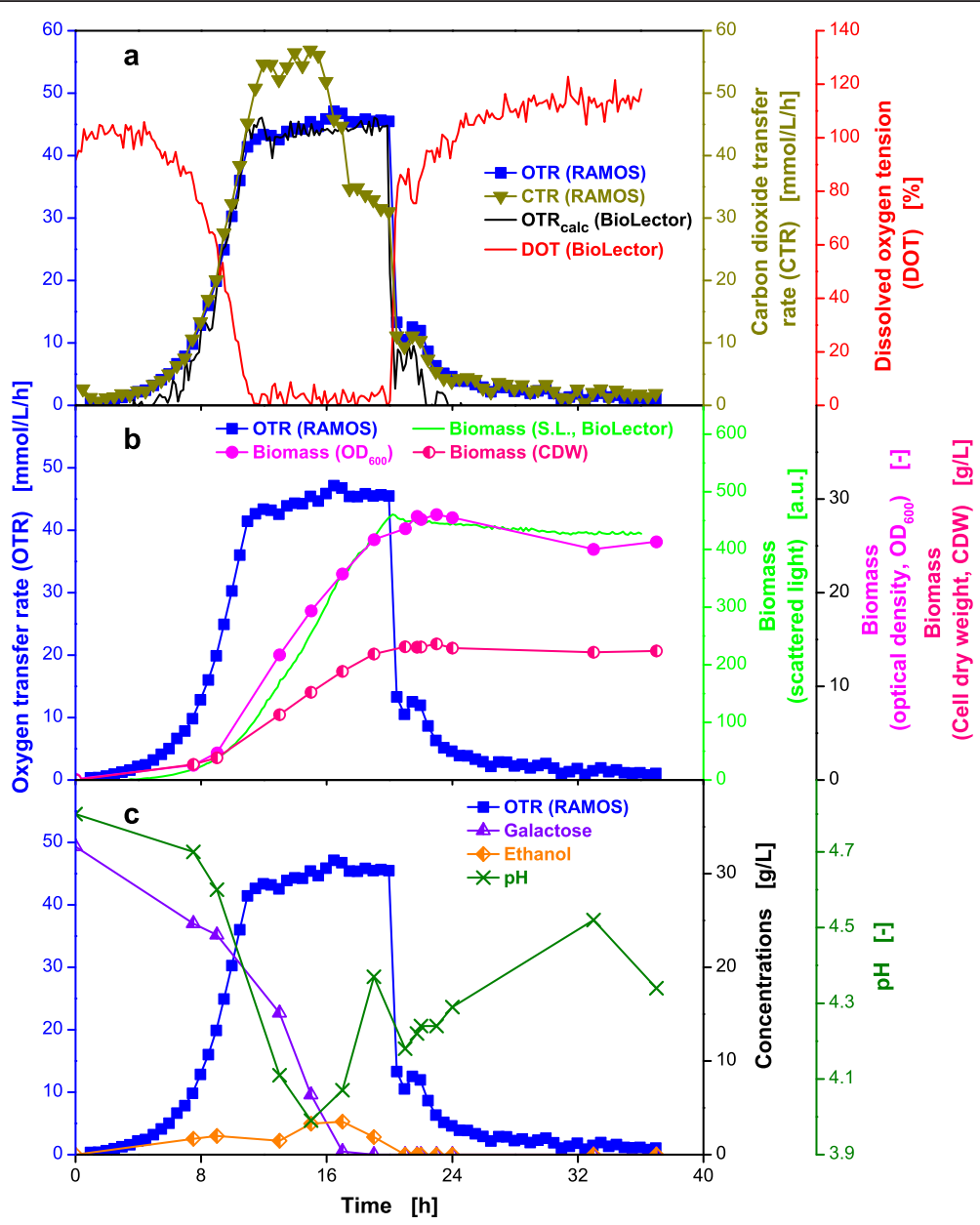

Fig. 8 Online and offline data from parallel fermentations of K. lactis Cel5A. Growth under oxygen-limited conditions in complex YEP-medium supplemented with $30 \mathrm{~g} / \mathrm{L}$ galactose (a-c). Culture conditions for RAMOS: $250 \mathrm{~mL}$ RAMOS shake flasks, $V_{\mathrm{L}}=20 \mathrm{~mL}, \mathrm{n}=350 \mathrm{rpm}, \mathrm{d}_{0}=50 \mathrm{~mm}$, $T=30^{\circ} \mathrm{C}$. Culture conditions for BioLector: 48-well Flowerplates, $V_{L}=1 \mathrm{~mL}, \mathrm{n}=800 \mathrm{rpm}, \mathrm{d}_{0}=3 \mathrm{~mm}, T=30^{\circ} \mathrm{C}$. The $\mathrm{k}_{\mathrm{L}} \mathrm{a}$ value used for calculation of the OTR calc course was $203 \mathrm{~h}^{-1}$ 
specific conditions. The offline data presented in Fig. 8c, show that galactose is used as carbon source and ethanol is produced simultaneously as previously described in literature [50]. The depletion of galactose is barely indicated by the OTR curve, whereby only a short small increase at $17 \mathrm{~h}$ is visible (Fig. 8a). However, the CTR curve indicates a drastic change in respiration behavior. After depletion of galactose and initiation of ethanol consumption, the CTR level drops from about 55 to $35 \mathrm{mmol} / \mathrm{L} / \mathrm{h}$, while the OTR level remains constant at $45 \mathrm{mmol} / \mathrm{L} / \mathrm{h}$. Depletion of ethanol causes a simultaneously drop in the OTR and CTR curve and the DOT value rises. Between 21 and $22 \mathrm{~h}$, the OTR and CTR curves show another shoulder which is mirrored by the DOT as well (Fig. 8a). Given the fact that no further secondary metabolites were detected in HPLC analysis, the cells supposedly grow on complex components of the YEP medium. Nevertheless, the carbon balance was closed to $99 \%$.

The decrease in the $\mathrm{pH}$-value during the first $16 \mathrm{~h}$ is attributed to the uptake of ammonium salts from the medium as stated in literature [51]. The subsequent rise and fall of the $\mathrm{pH}$-value can be explained by the uptake of complex proteinaceous components from the medium and the release of ammonium. Because the use of complex media makes a mechanistic interpretation of results much more difficult than results obtained with a defined mineral medium, one should avoid complex media. Complex media components resulting in high turbidity of the medium cause obvious problems when working with optical measurements.

\section{Conclusions}

This study has investigated the feasibility of a new experimental approach operating two culture- and onlinemonitoring devices based on shake flasks and microtiter plates (RAMOS and BioLector) in parallel to acquire comprehensive data on growth and production behavior of microorganisms. The obtained results are very helpful to understand complex metabolic relationships under different cultivation conditions such as diauxic growth, oxygen limitation and auto-induced product formation.

The operating conditions in shake flasks and microtiter plates can be precisely adjusted (for equivalent $\mathrm{OTR}_{\max }$ ) so that the microorganisms behave in an equivalent way over time. This conclusion is supported by the observation that the OTR in shake flasks and DOT signals in microtiter plates are mirror images of each other and the OTR calculated from the DOT measured in MTPs concurs with the measured OTR in in shake flasks. Additionally, the comparison of offline data from the MTP and shake flasks shows identical values demonstrating that equal conditions were applied during the cultivation. That the new approach is functional and effective could also be shown by the closed carbon balances. The preparation of one common mastermix for one experiment, before the inoculated medium is distributed among the different bioreactors, is an essential component of the new approach.

With this experimental technique problems like different lag-phases in the parallel running small-scale cultivation systems can be avoided and, therefore, we do not need to rely on calculating rates in the different systems. As four or even eight different experiments can be performed in parallel under very well controlled conditions, providing all the information usually obtained from laboratory-scale stirred tank bioreactors (equipped with an exhaust gas analyzer) the proposed experimental approach is very time and cost efficient. Therefore, the number of extensive experiments in laboratory-scale stirred tank bioreactors, related to strain selection and evaluation during initial process development (step 4 and 5 in Fig. 1), can be reduced by the suggested combination of smallscale culture systems.

The described approach is currently limited to batch experiments using $\mathrm{pH}$ buffers. However, it is imaginable to extend this approach also to fed-batch cultures, applying polymer based controlled release systems [52-55], enzymatic release systems (restricted to glucose) [56-62] or diffusion controlled feeding of compounds [63, 64]. With the first and last mentioned techniques also cultures with some degree of $\mathrm{pH}$ control at strongly reduced buffer concentration can be performed [64]. Additionally Funke et al. described a disposable and user-friendly microfluidic system for MTPs, which allows pH-controlled batch cultivations as well as fed-batch cultivations and show very good comparability with a laboratory-scale stirred tank bioreactor [28]. Investigations are currently performed to combine the aforementioned techniques for shake flasks and the herein presented new experimental approach, to make $\mathrm{pH}$-controlled and fed-batch cultivations feasible.

\section{Methods \\ Materials}

Chemicals were obtained from Sigma-Aldrich (Taufkirchen, Germany), Qiagen (Hilden, Germany), Merck (Darmstadt, Germany), Roth (Karlsruhe, Germany), and Roche Diagnostics (Mannheim, Germany).

\section{Strains}

In this study, four different strains were investigated: three bacterial strains, i.e., Escherichia coli BL21 (DE3) pRhotHi2-EcFbFP-His6 (E. coli BL21 EcFbFP), E. coli BL21 (DE3) pET22b(+)-His6-FbFP-BSLA-Ile12Cys (E. coli BL21 FbFP BSLA) and Gluconobacter oxydans DSM 3504, as well as a yeast strain, i.e., Kluyveromyces lactis Cel5A.

\section{Bacterial strains}

As cited above, two slightly different E. coli BL21 (DE3) strains were used. The first one expresses a FMN-based 
fluorescence protein (FbFP), codon-optimized for E. coli as expression host (hence, termed EcFbFP) $[65,66]$. This strain was kindly provided by Dr. Drepper from the group of Prof. Jaeger from the Institute for Molecular Enzyme Technology, Heinrich Heine University Düsseldorf.

The second strain, E. coli BL21 FbFP BSLA expresses Bacillus subtilis lipase A (BSLA) fused to FbFP and exhibits an amino acid exchange from isoleucine to cysteine at position 12 [65, 67]. This strain was also kindly provided by the group of Prof. Jaeger.

Gluconobacter oxydans DSM 3504 wild-type was kindly provided from the group of Prof. Liebl from the Technical University Munich, Department for Microbiology.

\section{Yeast strain}

Kluyveromyces lactis Cel5A was derived from the parental strain K. lactis GG799 which is a haploidic wild-type strain used in the food industry. The gene for the endocluconase Cel5A (EG III; UniProt-Nr.: P07982) was derived from Trichoderma reesei and cloned into $K$. lactis GG799 using the integrative vector pKLAC1. Data for product formation is not shown because it is not focus of this work. The strain was kindly provided from the group of Dr. Commandeur from RWTH Aachen, Institute for Molecular Biotechnology.

\section{Media}

\section{E. coli precultivation media}

To promote growth of $E$. coli, two precultivations with different media were performed. The first preculture was carried out in complex Terrific Broth (TB) [68] with the medium composed of $12 \mathrm{~g} / \mathrm{L}$ tryptone, $24 \mathrm{~g} / \mathrm{L}$ yeast extract, $12.54 \mathrm{~g} / \mathrm{L} \mathrm{K} \mathrm{KPO}_{4}, 2.3 \mathrm{~g} / \mathrm{L} \mathrm{K \textrm {K } _ { 2 }} \mathrm{PO}_{4}$ and $5 \mathrm{~g} / \mathrm{L}$ glycerol dissolved in deionized water. The $\mathrm{pH}$-value was $7.2 \pm 0.2$ without adjustment. Modified WilmsMOPS mineral medium was used for the second preculture and was prepared according to Wilms et al. [69]. The mineral medium consisted of $5 \mathrm{~g} / \mathrm{L}$ glycerol, $5 \mathrm{~g} / \mathrm{L}\left(\mathrm{NH}_{4}\right)_{2} \mathrm{SO}_{4}$, $0.5 \mathrm{~g} / \mathrm{L} \mathrm{NH}_{4} \mathrm{Cl}, 3 \mathrm{~g} / \mathrm{L} \mathrm{K}_{2} \mathrm{HPO}_{4}, 2 \mathrm{~g} / \mathrm{L} \mathrm{Na}_{2} \mathrm{SO}_{4}, 0.2 \mathrm{M}(\mathrm{N}-$ Morpholino)-propanesulfonic acid (MOPS), $0.5 \mathrm{~g} / \mathrm{L} \mathrm{MgSO}_{4}$. $7 \mathrm{H}_{2} \mathrm{O}, 0.01 \mathrm{~g} / \mathrm{L}$ thiamine hydrochloride, $1 \mathrm{~mL} / \mathrm{L}$ trace element solution $\left[0.54 \mathrm{~g} / \mathrm{L} \mathrm{ZnSO}{ }_{4} \cdot 7 \mathrm{H}_{2} \mathrm{O}, 0.48 \mathrm{~g} / \mathrm{L} \mathrm{CuSO}_{4}\right.$. $5 \mathrm{H}_{2} \mathrm{O}, 0.3 \mathrm{~g} / \mathrm{L} \quad \mathrm{MnSO}_{4} \cdot \mathrm{H}_{2} \mathrm{O}, 0.54 \mathrm{~g} / \mathrm{L} \quad \mathrm{CoCl}_{2} \cdot 6 \mathrm{H}_{2} \mathrm{O}$, $41.76 \mathrm{~g} / \mathrm{L} \mathrm{FeCl} \cdot 6 \mathrm{H}_{2} \mathrm{O}, 1.98 \mathrm{~g} / \mathrm{L} \mathrm{CaCl}{ }_{2} \cdot 2 \mathrm{H}_{2} \mathrm{O}, 33.4 \mathrm{~g} / \mathrm{L}$ $\mathrm{Na}_{2}$ EDTA (Titriplex III)]. The pH-value was adjusted to 7.5 using $1 \mathrm{M} \mathrm{NaOH}$. All medium components were sterilized separately by autoclaving or filtration before mixing. The concentration and type of $\mathrm{C}$-source is specified in the figure legends.

\section{E. coli main culture media}

Non-inducing growth conditions in mineral medium

For non-inducing growth conditions (non-oxygen-limited and-limited), the same modified WilmsMOPS mineral medium was used as described for the second precultivation in the section above. No glycerol was added; the respective concentrations of sugars are specified in the figure legends.

Inducing growth condition in mineral auto-induction medium If protein production ought to be induced, the previously described modified WilmsMOPS mineral medium for the second precultivation is supplemented with $0.5 \mathrm{~g} / \mathrm{L}$ glucose for initial growth and $2 \mathrm{~g} / \mathrm{L}$ sterilized lactose as inducing compound [70-72]. The glycerol concentration is kept at $5 \mathrm{~g} / \mathrm{L}$.

\section{Diauxic growth}

To examine diauxic growth behavior, modified WilmsMOPS mineral medium was prepared as mentioned for the second preculture but with altered substrate concentrations. No glycerol was added to the medium but rather a total of $20 \mathrm{~g} / \mathrm{L}$ glucose and $1.5 \mathrm{~g} / \mathrm{L}$ sorbitol.

\section{Gluconobacter medium}

The complex culture medium for G. oxydans DSM 3504 was prepared as described by Richardt et al. 2012 and was composed of $5 \mathrm{~g} / \mathrm{L}$ yeast extract, $1 \mathrm{~g} / \mathrm{L} \mathrm{KH}_{2} \mathrm{PO}_{4}$, $1 \mathrm{~g} / \mathrm{L}\left(\mathrm{NH}_{4}\right)_{2} \mathrm{SO}_{4}$ and $2.5 \mathrm{~g} / \mathrm{L} \mathrm{MgSO}_{4} 7 \mathrm{H}_{2} \mathrm{O}$ [73]. The $\mathrm{pH}$ was adjusted to 6.0 with $1 \mathrm{M} \mathrm{KOH}$. After sterilization at $121{ }^{\circ} \mathrm{C}$ for $21 \mathrm{~min}$, concentrated separately autoclaved glucose solution was added to obtain a final concentration of $40 \mathrm{~g} / \mathrm{L}$ glucose. Where indicated, the medium was supplemented with concentrated PIPPSbuffer solution to a final concentration of $100 \mathrm{mmol} / \mathrm{L}$.

\section{Kluyveromyces medium}

For the cultivation of $K$. lactis Cel5A, a basic complex YEP-medium was used. This consisted of $10 \mathrm{~g} / \mathrm{L}$ yeast extract and $20 \mathrm{~g} / \mathrm{L}$ peptone. The $\mathrm{pH}$-value was adjusted to 4.8 using $1 \mathrm{M} \mathrm{HCl}$ prior to sterilization at $121{ }^{\circ} \mathrm{C}$ for $21 \mathrm{~min}$. After the medium was allowed to cool down, concentrated separately autoclaved galactose solution was added to obtain a final concentration of $30 \mathrm{~g} / \mathrm{L}$ galactose.

\section{Cultivation parameters}

For all main cultivations, the so called 'mastermix' (medium with inoculated microrganisms) was prepared as follows: The medium for the main cultivation was made by adding the required supplements, and the beforehand determined volume of preculture to obtain the desired initial optical density.

\section{E. coli precultivations}

Precultivations of E. coli BL21 EcFbFP were performed in regular $250 \mathrm{~mL}$ non-baffled shake flasks. For the first preculture, $10 \mathrm{~mL}$ TB-medium containing $50 \mu \mathrm{g} / \mathrm{mL}$ 
kanamycin was inoculated with $100 \mu \mathrm{L}$ from a cryo stock (cell solution in $50 \%(\mathrm{w} / \mathrm{w})$ glycerol/water). Subsequently the non-baffled shake flasks were incubated overnight at a temperature $(\mathrm{T})$ of $37{ }^{\circ} \mathrm{C}$ on an orbital shaker at a shaking frequency (n) of $350 \mathrm{rpm}$ and a shaking diameter $\left(\mathrm{d}_{0}\right)$ of $50 \mathrm{~mm}$. As soon as an $\mathrm{OD}_{600}$ of $17 \pm 3$ was reached $(6-18 \mathrm{~h})$, the second preculture was started by inoculating $10 \mathrm{~mL}$ of modified WilmsMOPS mineral medium supplemented with $10 \mathrm{~g} / \mathrm{L}$ glucose to an initial $\mathrm{OD}_{600}$ of 0.1 . Cultivation parameters $\left(\mathrm{T}, \mathrm{n}, \mathrm{d}_{0}\right)$ were maintained as mentioned above, and the culture was incubated overnight for 8-11 h until an $\mathrm{OD}_{600}$ of $9 \pm$ 2 was obtained.

Precultivations for E. coli BL21 BSLA were carried out in modified $250 \mathrm{~mL}$ non-baffled shake flasks in an inhouse RAMOS device $[13,12]$ by applying a filling volume of $10 \mathrm{~mL}$. For incubation at $\mathrm{T}=37^{\circ} \mathrm{C}$, an orbital shaker was used with the shaking parameters $\mathrm{n}=$ $350 \mathrm{rpm}$ and $\mathrm{d}_{0}=50 \mathrm{~mm}$. For the first precultivation, $10 \mathrm{~mL}$ of $\mathrm{TB}$ medium containing $100 \mu \mathrm{g} / \mathrm{mL}$ ampicillin were inoculated with $100 \mu \mathrm{L}$ from a cryo stock (cell solution in $50 \%(\mathrm{w} / \mathrm{w})$ glycerol/water), incubated for $3 \mathrm{~h}$ and harvested as soon as an OTR value of $40-50 \mathrm{mmol} /$ $\mathrm{L} / \mathrm{h}$ had been reached. An aliquot of $10 \mathrm{~mL}$ of modified WilmsMOPS mineral medium supplemented with additional $0.5 \mathrm{~g} / \mathrm{L}$ glucose was inoculated with culture broth from the first precultivation to start the second precultivation. To achieve an initial optical density at $600 \mathrm{~nm}$ $\left(\mathrm{OD}_{600}\right)$ of 0.1 , the respective volume of culture broth from the first precultivation was added. Cultures were grown under the same incubation parameters $\left(\mathrm{T}, \mathrm{n}, \mathrm{d}_{0}\right)$ for $5 \mathrm{~h}$ and harvested at an OTR of $25-35 \mathrm{mmol} / \mathrm{L} / \mathrm{h}$.

\section{E. coli main cultivations}

Processing of the main cultures for both E. coli strains were identical. For all cultivations, a mastermix with an initial $\mathrm{OD}_{600}$ of 0.1 was prepared. Subsequently, the mastermix was divided among to the RAMOS flasks, microtiter plates and separate shake flasks (cultivation parameters are described in a later section). The respective volumes are specified in the figure legends.

\section{Cultivation of Gluconobacter oxydans}

Precultivations of G. oxydans were performed in $10 \mathrm{~mL}$ of the aforementioned complex medium in $250 \mathrm{~mL}$ nonbaffled shake flasks, without the addition of glucose. An antibiotic, $50 \mu \mathrm{g} / \mathrm{mL}$ cefoxitin sodium, was added prior to inoculation with $1 \mathrm{~mL}$ from a cryo stock (cell solution in $50 \%(\mathrm{w} / \mathrm{w})$ glycerol/water). The incubation was conducted overnight (12-14 h) on an orbital shaker at $30{ }^{\circ} \mathrm{C}$ with $\mathrm{n}=350 \mathrm{rpm}$ and $\mathrm{d}_{0}=50 \mathrm{~mm}$. After determination of the $\mathrm{OD}_{600}$, the required volume of cell broth to inoculate the main culture to an initial $\mathrm{OD}_{600}$ of 0.1 was calculated and transferred to a centrifugation tube to separate cells and medium. The harvested bacterial cells were re-suspended in fresh culture medium and subsequently transferred to the mastermix and distributed among RAMOS flasks, MTPs and separate shake flasks.

\section{Cultivation of Kluyveromyces lactis}

As preculture, an aliquot of $10 \mathrm{~mL}$ of YEP medium (without galactose) was filled into a $250 \mathrm{~mL}$ non-baffled shake flask and was inoculated with $100 \mu \mathrm{L}$ from a cryo stock (cell solution in $50 \%(\mathrm{w} / \mathrm{w})$ glycerol/water). After incubation at $30{ }^{\circ} \mathrm{C}$ with $\mathrm{n}=350 \mathrm{rpm}$ and $\mathrm{d}_{0}=50 \mathrm{~mm}$ for $12-14 \mathrm{~h}$ the cells were harvested at an $\mathrm{OD}_{600}$ value of $20 \pm 3$. Subsequently, the desired amount of culture volume was centrifuged, the supernatant was discarded, and the cell pellet was re-suspended in fresh YEP medium to an initial $\mathrm{OD}_{600}$ of 0.1 .

\section{Shake flask cultivations in the RAMOS device}

The in-house developed Respiration Activity MOnitoring System (RAMOS) $[12,13]$ allows online measurement of the oxygen transfer rate (OTR), carbon dioxide transfer rate (CTR) and of the respiratory quotient (RQ). Commercial versions of the RAMOS device are available from Kuhner AG, Birsfelden, Switzerland or HiTec Zang GmbH, Herzogenrath, Germany. The resulting characteristic curves provide information about growth and metabolic activity of the investigated microorganism. All cultivations were conducted with the individually specified culture conditions in $250 \mathrm{~mL}$ non-baffled shake flasks which were modified as described by Anderlei and Büchs [13].

\section{Microtiter plate cultivations in the BioLector device}

All microtiter plate (MTP) cultivations were performed in 48-well Flowerplates (m2p-labs, Baesweiler, Germany). For sterile cultivation conditions the plates were cover with an adhesive gas-permeable membrane (Thermo Scientific, Dreieich, Germany). An in-house developed BioLector device was used for non-invasive online measurement of scattered light (signal for biomass formation) and fluorescence (signal for product formation) during cultivations $[15,18]$. Commercial versions of the BioLector device are available from m2p-labs GmbH, Baesweiler, Germany. Signals were obtained by irradiating every single well with light of a defined wavelength (excitation), and detecting and analyzing the reflected/scattered light or fluorescence. In combination with special microtiter plates equipped with optodes on the bottom of the MTPs, dissolved oxygen tension (DOT) can be measured fluorometrically quasi-online and non-invasive. If not otherwise stated all BioLector cultivations were monitored with the adjustments summarized in the Table 1. 
Table 1 Overview of excitation and emission wavelengths in the BioLector

\begin{tabular}{lll}
\hline Signal & Filter (excitation/emission) & Gain \\
\hline E.coli BL21 EcFbFP & & 30 \\
Scattered light & $620 \mathrm{~nm} /-$ & 60 \\
FbFP-fluorescence & $450 \mathrm{~nm} / 492 \mathrm{~nm}$ & 60 \\
$\mathrm{pO}_{2}$-optode & $520 \mathrm{~nm} / 600 \mathrm{~nm}$ & 45 \\
$\mathrm{pH}$-optode & $470 \mathrm{~nm} / 525 \mathrm{~nm}$ & \\
E.coli BL21 BSLA & & 30 \\
Scattered light & $620 \mathrm{~nm} /-$ & 60 \\
FbFP-fluorescence & $450 \mathrm{~nm} / 492 \mathrm{~nm}$ & 60 \\
pO ${ }_{2}$-optode & $520 \mathrm{~nm} / 600 \mathrm{~nm}$ & \\
G. oxydans 3504 & & 30 \\
Scattered light & $620 \mathrm{~nm} /-$ & 50 \\
pO ${ }_{2}$-optode & $520 \mathrm{~nm} / 600 \mathrm{~nm}$ & \\
K. lactis Cel5A & & 30 \\
Scattered light & $620 \mathrm{~nm} /-$ & 50 \\
pO $_{2}$-optode & $505 \mathrm{~nm} / 590 \mathrm{~nm}$ & \\
\hline
\end{tabular}

\section{RoboLector system}

The BioLector device can be combined with various automated liquid handling systems ("pipetting robots"), which allows for experiment automation, including automated sampling [17]. Meanwhile this and comparable concepts have also been adapted by other research groups $[56,74,75]$.

\section{Shake flask cultivations}

Shake flask cultivations were carried out in conventional $250 \mathrm{~mL}$ non-baffled Erlenmeyer flasks with the respective filling volume (specified in figure legends) under identical cultivation conditions as described for the main cultivations. At different time points, single flasks were removed from the shaker and completely used for sampling. Carbon source and by-product concentrations were determined by HPLC. Moreover, $\mathrm{OD}_{600}$, cell dry weight and the $\mathrm{pH}$-value were measured offline.

\section{OTR $_{\text {max }}$ determination}

For determination of the maximum oxygen transfer capacity $\left(\mathrm{OTR}_{\max }\right)$ in shake flasks, the main culture of $E$. coli BL21 EcFbFP was grown on modified WilmsMOPS mineral medium containing $20 \mathrm{~g} / \mathrm{L}$ glucose and no glycerol. While keeping the shaking diameter $(50 \mathrm{~mm})$ and the shaking frequency $(350 \mathrm{rpm})$ constant, the filling volume was altered in a range of $8-25 \mathrm{~mL}$. The plateau

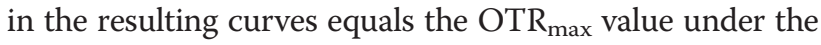
respective conditions (see Additional file 1: Figure S1).

In order to determine the maximum oxygen transfer capacity of a Flowerplate a modified RAMOS device was used which was first described 2005 by Kensy et al. [16].
The so-called MicroRAMOS is capable of measuring the oxygen transfer rate of a whole microtiter plate. The cultures were grown in WilmsMOPS mineral medium supplemented with $20 \mathrm{~g} / \mathrm{L}$ glucose and no glycerol. A high initial optical density $\left(\mathrm{OD}_{600}\right)$ of 1.0 was established to shorten the lag phase, thereby enabling the formation of multiple plateaus within one experimental run. For determining $\mathrm{OTR}_{\max }$, the shaking diameter was kept constant at $3 \mathrm{~mm}$ whereas the shaking frequency and the filling volume were altered in a range of 500-1000 rpm and $0.6-1.0 \mathrm{~mL}$, respectively. The attained plateaus represent the $\mathrm{OTR}_{\max }$ value of the applied cultivation condition (see Additional file 2: Figure S2).

\section{Software}

All calculations were performed with MATLAB (Version R2014a 8.3.0.532, The MathWorks, Inc., Natik, USA).

\section{Calculation of the oxygen solubility}

The oxygen solubilities for the respective cultivation media and temperatures were estimated according to Wilhelm et al. [76], Weisenberger \& Schumpe [77] and Rischbieter \& Schumpe [78]. Since the influence of Good's buffers was not investigated in these studies a hivalue of $0.2 \mathrm{~m}^{3} / \mathrm{kmol}$ for MOPS and PIPPS was assumed with respect to the molecular structure of investigated substances. Furthermore, the influence of sorbitol and galactose on the oxygen solubility was regarded as equal to the influence of glucose. Changes of the oxygen solubility during the cultivation due to changes of the media composition were not taken into account.

\section{Approximation of $k_{L} a$ values based on measured DOT and OTR}

Since the time vectors of the OTR measurements in the shake flask system (measurement point every $0.5 \mathrm{~h}$ ) and DOT measurements in the MTP (measurement point every $0.09 \mathrm{~h}$ ) were not identical, both time vectors were combined. Missing OTR and DOT values were interpolated with a cubic spline (MATLAB function: interp1, default settings). For each time point of the combined time vector corresponding values for OTR and DOT were available with these interpolated values. The oxygen transfer rate $\left(\mathrm{OTR}_{\text {calc }}\right)$ in MTPs is calculated from the measured DOTs according to the following equation:

$$
\mathrm{OTR}_{\text {calc }}=\mathrm{k}_{\mathrm{L}} \mathrm{a} \cdot \mathrm{L}_{\mathrm{O} 2} \cdot\left(\mathrm{pO}_{2}{ }^{\mathrm{gas}}-(\mathrm{DOT} / 100) \cdot \mathrm{pO}_{2}{ }^{\mathrm{cal}}\right)
$$

where $\mathrm{k}_{\mathrm{L}} \mathrm{a}\left[\mathrm{h}^{-1}\right]$ is the volumetric oxygen mass transfer coefficient, $\mathrm{L}_{\mathrm{O} 2}[\mathrm{~mol} / \mathrm{L} / \mathrm{bar}]$ is the oxygen solubility, $\mathrm{pO}_{2}{ }^{\text {gas }}$ [bar] is the oxygen partial pressure in the headspace of the MTP and $\mathrm{pO}_{2}{ }^{\mathrm{cal}}$ [bar] is the oxygen partial 
Table 2 Overview of the fitted $k_{L}$ a values

\begin{tabular}{|c|c|c|c|c|c|c|}
\hline \multirow[t]{3}{*}{ Cultivation } & \multirow[t]{3}{*}{$\mathrm{k}_{\mathrm{L}} \mathrm{a}\left[\mathrm{h}^{-1}\right]$} & \multirow{3}{*}{$\begin{array}{l}\text { Cultivation } \\
\text { temperature }\left[{ }^{\circ} \mathrm{C}\right]\end{array}$} & \multicolumn{4}{|c|}{ Shaking parameters } \\
\hline & & & \multicolumn{2}{|c|}{$\mathrm{MTP}^{1}$} & \multicolumn{2}{|c|}{ Shake flask ${ }^{2}$} \\
\hline & & & $\mathrm{n}$ [rpm] & $V_{L}[\mathrm{~mL}]$ & $\mathrm{n}$ [rpm] & $V_{L}[\mathrm{~mL}]$ \\
\hline E.coli non-oxygen-limited & 603 & 37 & 350 & 8 & 1500 & 1 \\
\hline E.coli oxygen-limited & 183 & 37 & 350 & 25 & 800 & 1 \\
\hline E.coli diauxic growth & 276 & 30 & 350 & 12 & 1000 & 0.6 \\
\hline E.coli induction & 587 & 37 & 350 & 10 & 1500 & 1 \\
\hline G. oxydans unbuffered & 425 & 30 & 350 & 10 & 1250 & 1 \\
\hline G. oxydans buffered & 413 & 30 & 350 & 10 & 1250 & 1 \\
\hline K. lactis oxygen-limited & 203 & 30 & 350 & 20 & 800 & 1 \\
\hline
\end{tabular}

1all culltivations performed at a shaking diameter of $3 \mathrm{~mm}$

2all culltivations performed at a shaking diameter of $50 \mathrm{~mm}$

pressure used for calibration of the DOT measurement system (0.21 bar).

For the present calculations an equal $\mathrm{OTR}_{\max }$ in MTPs and shake flasks is assumed for the compared conditions. Due to the intense ventilation of the culture chamber in the BioLector with air and the very high permeability of the applied sterile barriers (adhesive gaspermeable seal foils) [79] $\mathrm{pO}_{2}{ }^{\text {gas }}$ in the headspace of the MTP is assumed to be constant $(0.21$ bar $)$ throughout the whole cultivation. The $k_{L}$ a values for the MTP were approximated by the smallest sum of squared errors (method of least squares) between the above described measured OTR vector from shake flask and the DOTbased OTR calc values (Eq. 2) from MTP and are summarized in Table 2.

\section{Mass balance study}

Calculation of carbon balances have been performed applying Equation 3

$$
\mathrm{q}_{\mathrm{C}}^{\text {sub,i }}=\mathrm{q}_{\mathrm{C}}{ }^{\text {sub,r }}+\mathrm{q}_{\mathrm{C}}{ }^{\mathrm{CDW}}+\mathrm{q}_{\mathrm{C}}{ }^{\mathrm{CO} 2}+\mathrm{q}_{\mathrm{C}}{ }^{\mathrm{P}}
$$

where $\mathrm{q}_{C}{ }^{\text {sub,i }}$ is the molar concentration of carbon in the initial substrate, $\mathrm{q}_{C}{ }^{\text {sub,r }}$ of the residual substrate, $\mathrm{q}_{\mathrm{C}}{ }^{\mathrm{CDW}}$ of the cell dry weight, $\mathrm{q}_{C}{ }^{\mathrm{P}}$ of the product and $\mathrm{q}_{\mathrm{C}}{ }^{\mathrm{CO} 2}$ the calculated integral of the CTR. For E.coli and K. lactis a biomass composition of $\mathrm{C}_{1} \mathrm{H}_{1.8} \mathrm{O}_{0.5} \mathrm{~N}_{0.16}$ and $\mathrm{C}_{1} \mathrm{H}_{1.85}$ $\mathrm{O}_{0.574} \mathrm{~N}_{0.22}$ was assumed, respectively $[80,81]$.

\section{Offline analysis}

The dell dry weight (CDW) was determined gravimetrically. The $\mathrm{OD}_{600}$ of the cell broth was measured in a Genesys 20 photometer (Thermo Scientific, Dreieich, Germany).

HPLC analyses were performed using the UltiMate 3000 from Dionex, Germany. The metabolites were separated on an organic acid resin column $(250 \mathrm{~mm} \times$ $8 \mathrm{~mm}$ ) from CS-Chromatography Service (Langerwehe, Germany) at $60{ }^{\circ} \mathrm{C} .5 \mathrm{mM} \mathrm{H}_{2} \mathrm{SO}_{4}$ was used as eluent at a flow rate of $0.8 \mathrm{~mL} / \mathrm{min}$. A refractive index detector Shodex RI-101 (Shodwa Denko Europe, Germany) was used to detect and record the peaks.

For the samples from G. oxydans the same device and column was used but the analysis was performed at $80{ }^{\circ} \mathrm{C}$ using $1 \mathrm{mM} \mathrm{H}_{2} \mathrm{SO}_{4}$ as eluent at a flow rate of $0.8 \mathrm{~mL} \mathrm{~min}^{-1}$. Glucose concentrations in these samples were quantified via an enzymatic assay (Boehringer Mannheim, Germany). To adapt the assay procedure to a 96 well microtiter plate, only $10 \%$ of the specified volumes was used, reaching a target volume of $300 \mu \mathrm{L}$. The Synergy4 plate reader (BioTek Instruments, Bad Friedrichshall, Germany) was used to measure the absorbance at $340 \mathrm{~nm}$.

\section{Additional files}

Additional file 1: Figure S1. Determination of OTR max for E. coli BL21 EcFbFP in shake flasks. Cultivation in WilmsMOPS mineral medium supplemented with $20 \mathrm{~g} / \mathrm{L}$ glucose, buffered with $0.2 \mathrm{M}$ MOPS in a $250 \mathrm{~mL}$ RAMOS shake flasks, $V_{\mathrm{L}}=8-25 \mathrm{~mL}, \mathrm{n}=350 \mathrm{rpm}, \mathrm{d}_{0}=50 \mathrm{~mm}$, $\mathrm{T}=37^{\circ} \mathrm{C}$. All measurements were performed in duplicates.

Additional file 2: Figure S2. Determination of OTR $\max$ for E. coli BL21 EcFbFP in MTPs. Culture conditions for microRAMOS: WilmsMOPS mineral medium supplemented with $20 \mathrm{~g} / \mathrm{L}$ glucose, buffered with $0.2 \mathrm{M}$ MOPS, 48-well Flowerplates, $V_{L}=0.6-1 \mathrm{~mL}, \mathrm{n}=500-1000 \mathrm{rpm}, \mathrm{d}_{0}=3 \mathrm{~mm}$, $\mathrm{T}=37^{\circ} \mathrm{C}$. Arrows indicate the point at which the shaking frequency is shifted to the value written above.

Additional file 3: Figure S3. Determination of OTR max for E. coli BL21 EcFbFP in shake flasks. Cultivation in WilmsMOPS mineral medium supplemented with $10 \mathrm{~g} / \mathrm{L}$ glucose, buffered with $0.2 \mathrm{M} \mathrm{MOPS}$ in a $250 \mathrm{~mL}$ RAMOS shake flasks, $\mathrm{V}_{\mathrm{L}}=8-25 \mathrm{~mL}, \mathrm{n}=350 \mathrm{rpm}, \mathrm{d}_{0}=50 \mathrm{~mm}$, $\mathrm{T}=37^{\circ} \mathrm{C}$. All measurements were performed in duplicates.

\section{Abbreviations}

2-KGA: 2-ketogluconic acid; 5-KGA: 5-ketogluconic acid; a.u.: Arbitrary units; CTR: Carbon dioxide transfer rate; DOT: Dissolved oxygen tension; EcFbFP: E. coli modified FbFP; FbFP: FMN-based fluorescence protein; FMN: Flavin mononucleotide; $\mathrm{k}_{\mathrm{L}}$ a: Volumetric mass transfer coefficient; $\mathrm{L}_{\mathrm{O} 2}$ : Oxygen solubility in the medium; MOPS: 3-morpholinopropane-1sulfonic acid; MTP: Microtiter plate; OTR: Oxygen transfer rate;

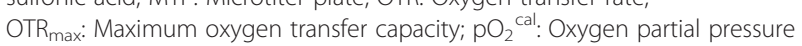
used for calibration; $\mathrm{PO}_{2}$ gas: Oxygen partial pressure in the headspace of the MTP; RAMOS: Respiration activity monitoring system; RQ: Respiration quotient; S.L.: Scattered light. 


\section{Competing interests}

The authors declare that they have no competing interests.

\section{Authors' contributions}

SW drafted the manuscript. MK designed this study. TL programmed the software to calculate the $k_{L} a$ and OTR values for the microtiter plate. RK performed cultivation experiments for non-limited, oxygen limited and diauxic growth. NR performed the cultivation of E. coli in auto-induction medium. BL designed the study of G. oxydans and supervised AS who conducted the cultivations. SR carried out the cultivations of K. lactis. LR co-supervised the study and assisted in drafting the manuscript. JB initiated and supervised the study and corrected the manuscript. All authors read and approved the final manuscript.

\section{Acknowledgements}

This work was performed as part of the Cluster of Excellence "Tailor-Made Fuels from Biomass", which is funded by the Excellence Initiative by the German federal and state governments to promote science and research at German universities.

\section{Author details}

${ }^{1}$ RWTH Aachen University, AVT - Biochemical Engineering, Worringer Weg 1 , 52074 Aachen, Germany. ${ }^{2}$ University of Minho, CEB - Centre of Biological Engineering, Campus de Gualtar, 4700-057 Braga, Portugal.

\section{Received: 5 December 2014 Accepted: 12 May 2015}

\section{Published online: 30 May 2015}

\section{References}

1. Bareither R, Pollard D. A review of advanced small-scale parallel bioreactor technology for accelerated process development: Current state and future need. Biotechnol Prog. 2011;27(1):2-14.

2. Gill NK, Appleton M, Baganz F, Lye GJ. Quantification of power consumption and oxygen transfer characteristics of a stirred miniature bioreactor for predictive fermentation scale-up. Biotechnol Bioeng. 2008;100(6):1144-55.

3. Gill NK, Appleton M, Baganz F, Lye GJ. Design and characterisation of a miniature stirred bioreactor system for parallel microbial fermentations. Biochem Eng J. 2008;39(1):164-76

4. Lee HLT, Boccazzi P, Ram RJ, Sinskey AJ. Microbioreactor arrays with integrated mixers and fluid injectors for high-throughput experimentation with pH and dissolved oxygen control. Lab Chip. 2006;6(9):1229-35.

5. Puskeiler R, Kaufmann K, Weuster-Botz D. Development, parallelization, and automation of a gas-inducing milliliter-scale bioreactor for high-throughput bioprocess design (HTBD). Biotechnol Bioeng. 2005;89(5):512-23.

6. Weuster-Botz D, Puskeiler R, Kusterer A, Kaufmann K, John GT, Arnold M. Methods and milliliter scale devices for high-throughput bioprocess design. Bioproc Biosyst Eng. 2005;28(2):109-19.

7. Klein T, Schneider K, Heinzle E. A system of miniaturized stirred bioreactors for parallel continuous cultivation of yeast with online measurement of dissolved oxygen and off-gas. Biotech Bioeng. 2013;110(2):535-42.

8. Vincentelli R, Cimino A, Geerlof A, Kubo A, Satou Y, Cambillau C. Highthroughput protein expression screening and purification in Escherichia coli. Methods. 2011;55(1):65-72.

9. Büchs J. Introduction to advantages and problems of shaken cultures. Biochem Eng J. 2001;7(2):91-8.

10. Maier U, Losen M, Büchs J. Advances in understanding and modeling the gas-liquid mass transfer in shake flasks. Biochem Eng J. 2004;17(3):155-67.

11. Amoabediny $G$, Büchs J. Modelling and advanced understanding of unsteady-state gas transfer in shaking bioreactors. Biotechnol Appl Biochem. 2007:46:57-67.

12. Anderlei T, Zang W, Papaspyrou M, Büchs J. Online respiration activity measurement (OTR, CTR, RQ) in shake flasks. Biochem Eng J. 2004;17(3):187-94.

13. Anderlei T, Büchs J. Device for sterile online measurement of the oxygen transfer rate in shaking flasks. Biochem Eng J. 2001;7(2):157-62.

14. Hermann R, Lehmann M, Büchs J. Characterization of gas-liquid mass transfer phenomena in microtiter plates. Biotechnol Bioeng. 2003:81(2):178-86.

15. Samorski M, Müller-Newen G, Büchs J. Quasi-continuous combined scattered light and fluorescence measurements: A novel measurement technique for shaken microtiter plates. Biotechnol Bioeng. 2005;92(1):61-8.
16. Kensy F, Zimmermann HF, Knabben I, Anderlei T, Trauthwein H, Dingerdissen $U$, et al. Oxygen transfer phenomena in 48-well microtiter plates: Determination by optical monitoring of sulfite oxidation and verification by real-time measurement during microbial growth. Biotechnol Bioeng. 2005;89(6):698-708.

17. Huber R, Ritter D, Hering T, Hillmer AK, Kensy F, Müller C, et al. Robo-Lector - a novel platform for automated high-throughput cultivations in microtiter plates with high information content. Microb Cell Fact. 2009;8:42-56.

18. Kensy F, Zang E, Faulhammer C, Tan RK, Büchs J. Validation of a highthroughput fermentation system based on online monitoring of biomass and fluorescence in continuously shaken microtiter plates. Microb Cell Fact. 2009;8:31-47.

19. Huber R, Roth S, Rahmen N, Büchs J. Utilizing high-throughput experimentation to enhance specific productivity of an E.coli $T 7$ expression system by phosphate limitation. BMC Biotechnol. 2011;11:22-32.

20. Huber R, Palmen TG, Ryk N, Hillmer AK, Luft K, Kensy F, et al. Replication methods and tools in high-throughput cultivation processes - recognizing potential variations of growth and product formation by on-line monitoring. BMC Biotechnol. 2010;10:22-31.

21. Kensy F, Engelbrecht C, Büchs J. Scale-up from microtiter plate to laboratory fermenter: Evaluation by online monitoring techniques of growth and protein expression in Escherichia coli and Hansenula polymorpha fermentations. Microb Cell Fact. 2009;8:68-82.

22. Guez JS, Müller CH, Danze PM, Büchs J, Jacques P. Respiration activity monitoring system (RAMOS), an efficient tool to study the influence of the oxygen transfer rate on the synthesis of lipopeptide by Bacillus subtilis ATCC6633. J Biotechnol. 2008;134(1-2):121-6.

23. Marques MPC, Cabral JMS, Fernandes P. Bioprocess scale-up: Quest for the parameters to be used as criterion to move from microreactors to lab-scale. J Chem Technol Biotechnol. 2010;85(9):1184-98.

24. Rocha-Valadez JA, Estrada M, Galindo E, Serrano-Carreon L. From shake flasks to stirred fermentors: Scale-up of an extractive fermentation process for 6 pentyl-alpha-pyrone production by Trichoderma harzianum using volumetric power input. Process Biochem. 2006;41(6):1347-52.

25. Seletzky JM, Noak U, Fricke J, Welk E, Eberhard W, Knocke C, et al. Scale-up from shake flasks to fermenters in batch and continuous mode with Corynebacterium glutamicum in lactic acid based on oxygen transfer and pH. Biotechnol Bioeng. 2007:98(4):800-11.

26. Trujillo-Roldán M, Valdez-Cruz N, Gonzalez-Monterrubio C, Acevedo-Sánchez E, Martínez-Salinas C, García-Cabrera R, et al. Scale-up from shake flasks to pilot-scale production of the plant growth-promoting bacterium Azospirillum brasilense for preparing a liquid inoculant formulation. Appl Microbiol Biotechnol. 2013;97(22):9665-74.

27. Micheletti M, Barrett T, Doig SD, Baganz F, Levy MS, Woodley JM, et al. Fluid mixing in shaken bioreactors: Implications for scale-up predictions from microlitre-scale microbial and mammalian cell cultures. Chem Eng Sci. 2006;61(9):2939-49.

28. Funke M, Buchenauer A, Mokwa W, Kluge S, Hein L, Mueller C, et al. Bioprocess control in microscale: Scalable fermentations in disposable and user-friendly microfluidic systems. Microb Cell Fact. 2010;9:86-98.

29. Islam RS, Tisi D, Levy MS, Lye GJ. Scale-up of Escherichia coli growth and recombinant protein expression conditions from microwell to laboratory and pilot scale based on matched kLa. Biotechnol Bioeng. 2008;99(5):1128-39.

30. Britten R. Extracellular metabolic products of Escherichia coli during rapid growth. Science. 1954;119(3096):578-69.

31. Nyström T. Stationary-phase physiology. Annu Rev Microbiol. 2004;58:161-81.

32. Siepert EM, Gartz E, Tur MK, Delbrück H, Barth S, Büchs J. Short-chain fluorescent tryptophan tags for on-line detection of functional recombinant proteins. BMC Biotechnol. 2012;12:65-77.

33. Kottmeier K, Weber J, Müller C, Bley T, Büchs J. Asymmetric division of Hansenula polymorpha reflected by a drop of light scatter intensity measured in batch microtiter plate cultivations at phosphate limitation. Biotechnol Bioeng. 2009;104(3):554-61.

34. Losen M, Frölich B, Pohl M, Büchs J. Effect of oxygen limitation and medium composition on Escherichia coli fermentation in shake-flask cultures. Biotechnol Prog. 2004;20(4):1062-8.

35. Zimmermann HF, Anderlei T, Büchs J, Binder M. Oxygen limitation is a pitfall during screening for industrial strains. Appl Microbiol Biotechnol. 2006;72(6):1157-60.

36. Lorantfy $B$, Jazini M, Herwig C. Investigation of the physiological response to oxygen limited process conditions of Pichia pastoris Mut(+) strain using a two-compartment scale-down system. J Biosci Bioeng. 2013;116(3):371-9. 
37. Xu B, Jahic M, Enfors SO. Modeling of overflow metabolism in batch and fed-batch cultures of Escherichia coli. Biotechnol Prog. 1999;15(1):81-90.

38. Hansen S, Hariskos I, Luchterhand B, Büchs J. Development of a modified Respiration Activity Monitoring System for accurate and highly resolved measurement of respiration activity in shake flask fermentations. J Biol Eng. 2012;6(1):11-22.

39. Monod J. The growth of bacterial cultures. Annu Rev Microbiol. 1949;3:371-94.

40. Krahe M, Antranikian G, Märkl H. Fermentation of extremophilic microorganisms. Fems Microbiol Rev. 1996;18(2-3):271-85.

41. Inada T, Kimata K, Aiba HJ. Mechanism responsible for glucose-lactose diauxie in Escherichia coli: Challenge to the cAMP model. Genes Cells. 1996;1(3):293-301.

42. Bentley WE, Mirjalili N, Andersen DC, Davis RH, Kompala DS. Plasmidencoded protein: The principal factor in the "Metabolic Burden" associated with recombinant bacteria. Biotechnol Bioeng. 2009;102(5):1284-97.

43. Neubauer P, Lin HY, Mathiszik B. Metabolic load of recombinant protein production: Inhibition of cellular capacities for glucose uptake and respiration after induction of a heterologous gene in Escherichia coli. Biotechnol Bioeng. 2003;83(1):53-64.

44. Kunze M, Huber R, Gutjahr C, Müllner S, Büchs J. Predictive tool for recombinant protein production in Escherichia coli shake-flask cultures using an on-line monitoring system. Biotechnol Prog. 2012;28(1):103-13.

45. Rahmen N, Fulton A, Ihling N, Magni M, Jaeger KE, Büchs J. Exchange of single amino acids at different positions of a recombinant protein affects metabolic burden in Eschericha coli. Microb Cell Fact. 2015;10(14):1-18.

46. De Muynck C, Pereira CSS, Naessens M, Parmentier S, Soetaert W, Vandamme EJ. The genus Gluconobacter oxydans: Comprehensive overview of biochemistry and biotechnological applications. Crit Rev Biotechnol. 2007:27(3):147-71.

47. Elfari M, Ha SW, Bremus C, Merfort M, Khodaverdi V, Herrmann U, et al. A Gluconobacter oxydans mutant converting glucose almost quantitatively to 5-keto-D-gluconic acid. Appl Microbiol Biotechnol. 2005;66(6):668-74.

48. Kunze M, Roth S, Gartz E, Büchs J. Pitfalls in optical on-line monitoring for high-throughput screening of microbial systems. Microb Cell Fact. 2014;13:53-72.

49. Luchterhand B, Fischöder T, Grimm AR, Wewetzer S, Wunderlich M, Schlepütz T, et al. Quantifying the sensitivity of G. oxydans ATCC $621 \mathrm{H}$ and DSM 3504 to osmotic stress triggered by soluble buffers. J Ind Microbiol Biotechnol. 2015;42(4):585-600.

50. González Siso MI, Ramil E, Cerdán ME, Freire-Picos MA. Respirofermentative metabolism in Kluyveromyces lactis: Ethanol production and the Crabtree effect. Enzyme Microb Technol. 1996;18(8):585-91.

51. Christensen ML, Eriksen NT. Growth and proton exchange in recombinant Escherichia coli BL21. Enzyme Microb Technol. 2002;31(4):566-74.

52. Huber R, Scheidle M, Dittrich B, Klee D, Büchs J. Equalizing growth in highthroughput small-scale cultivations via precultures operated in fed-batch mode. Biotechnol Bioeng. 2009;103(6):1095-102.

53. Scheidle M, Jeude M, Dittrich B, Denter S, Kensy F, Suckow M, et al. Highthroughput screening of Hansenula polymorpha clones in the batch compared with the controlled-release fed-batch mode on a small scale. Fems Yeast Research. 2010;10(1):83-92.

54. Jeude M, Dittrich B, Niederschulte H, Anderlei T, Knocke C, Klee D, et al. Fed-batch mode in shake flasks by slow-release technique. Biotech Bioeng. 2006;95(3):433-45.

55. Scheidle M, Dittrich B, Bähr C, Büchs J. Freisetzungssysteme zur Prozessentwicklung in Kleinkulturen. BIOspektrum. 2013;19(1):96-8.

56. Hemmerich J, Adelantado N, Barrigón JM, Ponte X, Hörmann A, Ferrer P, et al. Comprehensive clone screening and evaluation of fed-batch strategies in a microbioreactor and lab scale stirred tank bioreactor system: Application on Pichia pastoris producing Rhizopus oryzae lipase. Microb Cell Fact. 2014;13:36-51.

57. Panula-Perälä J, Šiurkus J, Vasala A, Wilmanowski R, Casteleijn MG, Neubauer P. Enzyme controlled glucose auto-delivery for high cell density cultivations in microplates and shake flasks. Microb Cell Fact. 2008;7

58. Šiurkus J, Panula-Perälä J, Horn U, Kraft M, Rimšeliene R, Neubauer P. Novel approach of high cell density recombinant bioprocess development: Optimisation and scale-up from microlitre to pilot scales while maintaining the fed-batch cultivation mode of E. coli cultures. Microb Cell Fact. 2010;9.

59. Šiurkus J, Neubauer P. Reducing conditions are the key for efficient production of active ribonuclease inhibitor in Escherichia coli. Microb Cell Fact. 2011;10
60. Šiurkus J, Neubauer P. Heterologous production of active ribonuclease inhibitor in Escherichia coli by redox state control and chaperonin coexpression. Microb Cell Fact. 2011;10.

61. Krause M, Ukkonen K, Haataja T, Ruottinen M, Glumoff T, Neubauer A, et al. A novel fed-batch based cultivation method provides high cell-density and improves yield of soluble recombinant proteins in shaken cultures. Microb Cell Fact. 2010;9

62. Glazyrina J, Krause M, Junne S, Glauche F, Strom D, Neubauer P. Glucose-limited high cell density cultivations from small to pilot plant scale using an enzymecontrolled glucose delivery system. New Biotechnol. 2012;29(2):235-42.

63. Bähr C, Leuchtle B, Lehmann C, Becker J, Jeude M, Peinemann F, et al. Dialysis shake flask for effective screening in fed-batch mode. Biochem Eng J. 2012;69:182-95.

64. Wilming A, Bähr C, Kamerke C, Büchs J. Fed-batch operation in special microtiter plates: A new method for screening under production conditions. J Ind Microbiol Biotechnol. 2014;41(3):513-25.

65. Drepper T, Eggert T, Circolone F, Heck A, Krauss U, Guterl JK, et al. Reporter proteins for in vivo fluorescence without oxygen. Nat Biotechnol. 2007;25(4):443-5.

66. Drepper T, Huber R, Heck A, Circolone F, Hillmer AK, Büchs J, et al. Flavin mononucleotide-based fluorescent reporter proteins outperform green fluorescent protein-like proteins as quantitative in vivo real-time reporters. Appl Environ Microbiol. 2010;76(17):5990-4.

67. Krauss U, Lee J, Benkovic SJ, Jaeger KE. LOVely enzymes - towards engineering light-controllable biocatalysts. Microb Biotechnol. 2010;3(1):15-23.

68. Tartoff KD, Hobbs CA. Improved media for growing plasmid and cosmid clones. Bethesda Res Lab Focus. 1987;9(2):1.

69. Wilms B, Hauck A, Reuss M, Syldatk C, Mattes R, Siemann M, et al. High-celldensity fermentation for production of L-N-carbamoylase using an expression system based on the Escherichia coli rhaBAD promoter. Biotechnol Bioeng. 2001;73(2):95-103.

70. Studier FW. Protein production by auto-induction in high-density shaking cultures. Protein Expr Purif. 2005;41(1):207-34.

71. Blommel PG, Becker KJ, Duvnjak P, Fox BG. Enhanced bacterial protein expression during auto-induction obtained by alteration of lac repressor dosage and medium composition. Biotechnol Prog. 2007;23(3):585-98.

72. Li ZP, Kessler W, van den Heuvel J, Rinas U. Simple defined autoinduction medium for high-level recombinant protein production using T7-based Escherichia coli expression systems. Appl Microbiol Biotechnol. 2011;91(4):1203-13.

73. Richhardt J, Luchterhand B, Bringer S, Büchs J, Bott M. Evidence for a key role of cytochrome bo(3) oxidase in respiratory energy metabolism of Gluconobacter oxydans. J Bacteriol. 2013;195(18):4210-20.

74. Rohe P, Venkanna D, Kleine B, Freudl R, Oldiges M. An automated workflow for enhancing microbial bioprocess optimization on a novel microbioreactor platform. Microb Cell Fact. 2012;11

75. Hemmerich J, Kensy F. Automated microbioreactor systems for pharmaceutical bioprocessing: Profiling of seeding and induction conditions in high-throughput fermentations. Pharm Bioprocess. 2014;2(3):227-35.

76. Wilhelm E, Battino R, Wilcock RJ. Low-pressure solubility of gases in liquid water. Chem Rev. 1977;77(2):219-62.

77. Weisenberger S, Schumpe A. Estimation of gas solubilities in salt solutions at temperatures from 273 K to 363 K. AlChE J. 1996;42(1):298-300.

78. Rischbieter E, Schumpe A, Wunder V. Gas solubilities in aqueous solutions of organic substances. J Chem Eng Data. 1996;41(4):809-12.

79. Zimmermann HF, John GT, Trauthwein H, Dingerdissen U, Huthmacher K. Rapid evaluation of oxygen and water permeation through microplate sealing tapes. Biotechnol Prog. 2003;19(3):1061-3.

80. Kayser A, Weber J, Hecht V, Rinas U. Metabolic flux analysis of Escherichia coli in glucose-limited continuous culture. I. Growth-ratedependent metabolic efficiency at steady state. Microbiol SGM. 2005;151:693-706.

81. Herwig C, von Stockar U. Quantitative comparison of transient growth of Saccharomyces cerevisiae, Saccharomyces kluyveri, and Kluyveromyces lactis. Biotechnol Bioeng. 2003;81(7):837-47. 